\title{
On the Quantum Entanglement Reinterpretation Using the Time as Real Instantaneous Signal Field
}

\author{
Elsadig Naseraddeen \\ Founder and General Manager, Information Fluxes-FZE, Dubai, UAE \\ Email: s.naseraddeen@hotmail.com
}

How to cite this paper: Naseraddeen, E. (2017) On the Quantum Entanglement Reinterpretation Using the Time as Real Instantaneous Signal Field. Journal of Modern Physics, 8, 853-891.

https://doi.org/10.4236/jmp.2017.86054

Received: January 14, 2017

Accepted: May 15, 2017

Published: May 18, 2017

Copyright $\odot 2017$ by author and Scientific Research Publishing Inc. This work is licensed under the Creative Commons Attribution International License (CC BY 4.0).

http://creativecommons.org/licenses/by/4.0/

\begin{abstract}
In relativistic mechanics the time-like vector characterize the motion in spacetime with speed faster than the speed of light in vacuum $c$ in which the line element $d s^{2}=c^{2} d t^{2}-d x^{2}-d x^{2}-d z^{2}$ is less than zero (where $d t$ is infinitesimal change in time, $d x, d y$ and $d z$ are infinitesimal change in space), thus the time in relativistic mechanics can instantaneously flow [1], however in quantum mechanics although the time is treated as unobservable parameter (without any Hermitian observable operator have engine-value equivalent to time) any two physical quantity described by two non-commuting observable operators $\hat{A}$ and $\hat{B}$ fulfill $\hat{A} \hat{B} \neq \hat{B} \hat{A}$, the knowledge of one immediately produce the knowledge of the other [2], thus in quantum mechanics if two particles interacted in finite temporal epoch and then separated in space the gaining of knowledge by the local measurement of physical quantity runs on one them (for example the measurement of spin direction of one particle using Stern-Gerlach experiment) immediately produce the knowledge of the complementary physical quantity of the other particle (for example the opposite spin direction of the other particle), this simply called quantum entanglement the concept that so much advanced after publication of the Jon Bell's 1964 celebrated paper [3] in which he illustrated that we can add parameters to quantum mechanics to determine the results of individual measurements, without changing the statistical predictions, and then he conclude "there must be a mechanism whereby the setting of one measuring device can influence the reading of another instrument, however remote. Moreover, the signal involved must propagate instantaneously so that such a theory could not be Lorentz invariant'. The question now what these signals that can propagate instantaneously? The answer in this paper will be the time signals field which is defined for each constituent matter particle $M$ and at each space point $P$ as the measure of the total length of all occupation and leaving epochs of $P$ by $M$ which is representing a sequence function compactly sup-
\end{abstract}


ported only at the space point occupied by it and indexed by the number of occupation epochs of $P$ by $M$, thus the flow of this time signal field from the far future to near future through the present to the near past to the far past inferable by the flow of matter particles constituting the system(such as sun, moon earth and clocks hands). Thus the present will represent in this paper a local absolute feature of time signals field defined at each space point as the set of all occupation epochs of it by matter particle, however the past and future will represent relativistic non-local features of the time signal field defined at each space point as a set of all leaving epochs between each two sequential occupation epochs, so the future after one occupation epoch is representing a past of the next one. Thus according to current representation of time, the two Mc-Taggard's A and B series of time [4] will exist together as temporal set and then the time is real, the A-series in current theory is a set of all occupation and leaving epochs of space point by the matter particle that is consisting of the present, past and future epochs, and the B-series is the set of all leaving epochs of space points between each two sequential occupation epochs which are taking position before or after the discrete occupation epochs between them and then before or after each other.

\section{Keywords}

Real-Time State, Real-Time Digital State, Real-Time Transition State, Entanglement Translation, Digital Levels, Calculus of Fluctuations, Fluctuation Tensor Field, Collinear Space, Coplanar Space

\section{Introduction}

The direction of using the quantum entanglement in the measurement of time introduced by Don Page and Wooster who are argued that quantum entanglement can be used to measure time [5], other theorist used quantum entanglement to explain the flow of time [6]. The current investigation of time using the quantum entanglement will take new direction in which we can represent the real time state of any physical system consisting of one or more matter particles at any space point $P$ as single entangled state called hereinafter the real-time state with dimension equivalent to the number of constituent matter particles of the physical system and with components equivalent to time of each one of them at $P$, the author will investigate the translation of this real-time state as quantum entanglement phenomena in which the measurement of occupation of $P$ by any one constituent matter particle of the physical system $S$ immediately produce the equivalent measurement information of the part of lengths of all leaving epochs of $P$ by the rest constituent matter particles of $S$ that occupied and left $P$, this translation as we will see implied the existence of finite set of digital states which are: 1) representing a basis for Entanglement Translation of the real-time state at each spatial position, and 2) distributed into set of sequential digital levels in which the real-time state of the physical system is transits from one digital level to the next digital level equivalently to the orbital transition. 


\section{Basis Formulation}

\subsection{Why We Need for the Current Theory of Space and Time}

In order to understand why we need to the current theory of time take for example the mechanism of forwarding the time in the analog clock, in which all three hands-second, minute and hour hand-are occupying and leaving their occupies space when the impulse system of the analog clock exerting a single impulse acting on all of them simultaneously, this single impulse is representing superposition of all electromagnetic wave that are reflecting by the surface's points of underlying elementary constituent matter particle of the clock's hands and its remainder parts at each exerting epoch of impulse system, however there is existing strong correlation between the number of exerted impulses, the number of occupation epochs of points in the space of motion of the clock's hands by them and the measurable time by tracking the paths occupied by the analog clock's hands, we can illustrating this correlation using the fact that the duration of each impulse is one second to write the time $t$ at each point in the space of motion of the specified clock's hand $\mathrm{CH}$ of this analog clock defined with respect to the join points of its hands by $(x, y, z)$ during or after the $n^{\text {th }}$ occupation epoch of $(x, y, z)$ by $C H$ as the sequence function

$$
\begin{aligned}
t & (x, y, z, n)=t_{0}(x, y, z)+\sum_{m=1}^{n-1} I(x, y, z, m) \\
& +\sum_{m=1}^{n-1}(T(x, y, z, m)-I(x, y, z, m))+I(x, y, z, n)+\delta t_{n}(x, y, z) \\
= & t_{0}(x, y, z)+\sum_{m=1}^{n-1}[T(x, y, z, m)]+I(x, y, z, n)+\delta t_{n}(x, y, z) \\
= & t_{0}(x, y, z)+(n-1) \bar{T}(x, y, z, m)+I(x, y, z, n)+\delta t_{n}(x, y, z)
\end{aligned}
$$

seconds, such that $t_{0}(x, y, z)$ is initial leaving epoch of $(x, y, z)$ started when the observer starting the tracking of the motion of $\mathrm{CH}$ and ending at starting of the first occupation epoch of $(x, y, z)$ by $C H, I(x, y, z, k)$ for all $k \in\{1,2, \cdots, n\}$ is representing the number of impulses exerted on $C H$ by the analog clock's impulse system during the $k^{\text {th }}$ occupation epoch of $(x, y, z)$ by $C H, T(x, y, z, m)$ for all $m \in\{1,2, \cdots, n-1\}$ is representing the summation of the number of impulses exerting after the time $t_{0}(x, y, z)$ on $C H$ during the $m^{\text {th }}$ occupation epoch of $(x, y, z)$ by it and the number of impulses exerted on it during the $m^{\text {th }}$ leaving epoch of $(x, y, z)$ by it, and $\bar{T}(x, y, z, n)$ is average of $T(x, y, z, 1), T(x, y, z, 2), \cdots$ and $T(x, y, z, n-1)$ fulfills $(n-1) \bar{T}(x, y, z, m)=\sum_{m=1}^{n-1} T(x, y, z, m)$, thus $\sum_{m=1}^{n-1} I(x, y, z, m)$ is representing the total lengths of all first $n-1$ occupation epochs of $(x, y, z)$ by $C H$ after the time $t_{0}(x, y, z), \sum_{m=1}^{n-1}(T(x, y, z, m)-I(x, y, z, m))$ is representing the total length of all first $n-1$ leaving epochs of $(x, y, z)$ by $C H$ occurred between each two occupation epochs of $(x, y, z)$ by it after the initial time $t_{0}(x, y, z)$ and $\delta t_{n}(x, y, z)$ is the length of the leaving epoch of $(x, y, z)$ by $C H$ elapsed after the end of the $n^{\text {th }}$ occupation epoch of $(x, y, z)$ by it, so if the analog clock is ideally perfect then $T(x, y, z, m)=\bar{T}(x, y, z, m)=60,3600$ or 216,000 in a case of $t(x, y, z, n)$ is representing the observable time measurable by tracking the motion second, minute or hour hand respectively, now if we have $N$ identical perfect analog clocks such that for every $i \in\{1,2, \cdots, N\}$ the point $(x, y, z)$ is 
representing a point in the space of motion of the specified hand of the $i^{\text {th }}$ analog clock defined with respect to the join points of its hands, $n_{i}$ is representing the number of occupation epochs of $(x, y, z)$ by $\mathrm{CH}_{i}$-which is clock hand of the $I^{\text {th }}$ analog clock that we took under consideration, $I_{i}(x, y, z, k)$ for each

$k=1,2, \cdots, n_{i}$, is representing the number of impulses exerted on it by the impulse system of the $i^{\text {th }}$ analog clock during the $k^{\text {th }}$ occupation epoch of $(x, y, z)$ by $C H_{i}, T_{i}(x, y, z, m)$ for all $m \in\left\{1,2, \cdots, n_{i}-1\right\}$ is representing the summation of the number of impulses exerting on $\mathrm{CH}_{i}$ during the $\mathrm{m}^{\text {th }}$ occupation epoch of $(x, y, z)$ by it and the number of impulses exerting on it during the $m^{\text {th }}$ leaving epoch of $(x, y, z)$ by it, and $\bar{T}_{i}\left(x, y, z, n_{i}\right)$ is average of $T_{i}(x, y, z, 1), T_{i}(x, y, z, 2), \cdots$ and $T_{i}\left(x, y, z, n_{i}-1\right)$ fulfills $\left(n_{i}-1\right) \bar{T}_{i}\left(x, y, z, n_{i}\right)=\sum_{m=1}^{n_{i}-1} T_{i}(x, y, z, m)$ then we can define the real time state of this $N$ analog clocks by

$t=t\left(x, y, z, n_{1}, n_{2}, \cdots, n_{N}\right)=\left(t_{1}\left(x, y, z, n_{1}\right), t_{2}\left(x, y, z, n_{2}\right), \cdots, t_{N}\left(x, y, z, n_{N}\right)\right)$, such that $t_{i}\left(x, y, z, n_{i}\right)=t_{0}^{i}(x, y, z)+\left(n_{i}-1\right) \bar{T}_{i}\left(x, y, z, n_{i}\right)+I_{i}\left(x, y, z, n_{i}\right)+\delta t_{n_{i}}(x, y, z)$ seconds where $t_{0}^{i}(x, y, z)$ is initial time elapsed during the epoch started when the observer starting the tracking of $\mathrm{CH}_{i}$ and ending at starting of the first occupation epoch of $(x, y, z)$ by it and $\delta t_{n_{i}}(x, y, z)$ is the length of leaving epoch of $(x, y, z)$ by $C H_{i}$ elapsed after the end of the $n_{i}^{\text {th }}$ occupation epoch of $(x, y, z)$ by it, now according to the classical mechanics the time is absolute at all occupation and leaving epochs of epoch of $(x, y, z)$ by $\mathrm{CH}_{i}$ and then if $t_{0}^{i}=t_{0}^{j}$ for all $i, j \in\{1,2, \cdots, N\}$ then all second, minute and hour hands of these identical clocks are synchronously occupy their corresponding points in their spaces of motion and synchronously leave them regardless of their spatial distribution or their surfaces orientation in space, and hence the real time state $t$ according to classical mechanics should alwayslied at the equilibrium collinear set $\overline{\overline{\mathbb{R}^{N}}}=\left\{\left(t_{1}, t_{2}, \cdots, t_{N}\right) \in \mathbb{R}^{N} \mid t_{j}=t_{i}\right.$ for every $\left.i, j \in\{1,2, \cdots, N\}\right\}$, however in general relativity Einstein followed another direction and argued the existence of what is now called the gravitation time dilation [1] which implied that the gravitation field have different value of stress-energy-momentum tensor in different space points occupies by different hands of these analog clocks cause their running at different rates, so according to the general theory of relativity the real time state $t$ may deviating from the equilibrium collinear set $\overline{\overline{\mathbb{R}^{N}}}$ as result of difference in gravitation field from one point of space to another point, thus we need mathematical formulation provide a measure to degree to which the real time state of any quantum system consisting of $N$ matter Particles at any points inside their occupies paths from the equilibrium collinear set $\overline{\overline{\mathbb{R}^{N}}}$ and all another non-equilibrium collinear set in $\mathbb{R}^{N}$, the author will approve that each equilibrium or non-equilibrium collinear set is representing vector subspace of $\mathbb{R}^{N}$, and then if this vector subspaces endowed with usual dot product they will represent vector subspaces of $\mathrm{n}$-dimensional Euclidian space which is representing an inner product Hilbert space.

\subsection{The Occupation Epoch Number of the Matter Particle}

The occupation epoch number of the matter particle at each space point $(x, y, z)$ 
donated by $n(x, y, z)$ is representing the number of occupation epochs of $(x, y, z)$ by the matter particle with respect to some observer or measurement instrument observing the motion of matter particle during finite observation epoch.

\subsection{The Time of the Matter Particle at Each Space Point}

If $n=n(x, y, z)$ is the occupation epoch number of the matter particle $P$ at the space point $(x, y, z)$ then the time of the matter particle $P$ at the space point $(x, y, z)$ is defined as the total length of all the occupation and leaving epochs of $(x, y, z)$ by the matter particle $P$ and then is defined as following:

$$
\begin{gathered}
t(x, y, z, n)=\delta t(x, y, z, 0)+(n-1) \bar{T}(x, y, z, n)+d t(x, y, z, n)+\delta t(x, y, z, n)(1) \\
\bar{T}(x, y, z, n)= \begin{cases}d t(x, y, z, 1)+\delta t(x, y, z, 1), & n=1 \\
\frac{1}{n-1} \sum_{i=1}^{n-1}[d t(x, y, z, i)+\delta t(x, y, z, i)], & n>1 \\
0, & n=0\end{cases}
\end{gathered}
$$

where: $\delta t(x, y, z, 0)$ is the length of the initial leaving epoch elapsed before the first occupation of $x, y, z$ by the matter particle with respect to some observer or measurement instrument observing the motion of matter particle during finite observation epoch.

For each $i \in\{1,2, \cdots, n-1\}$ :

$d t(x, y, z, i)$ is the length of the $I^{\text {th }}$ occupation epoch of $x, y, z$ by the matter particle.

$\delta t(x, y, z, i)$ is the length of the $i^{\text {th }}$ leaving epoch of $x, y, z$ by the matter particle elapsed aft the $i^{\text {th }}$ occupation epoch.

$d t(x, y, z, n)$ is the length of the $n^{\text {th }}$ occupation epoch of $x, y, z$ by the matter particle.

$\delta t(x, y, z, n)$ is the length of the epoch elapsed after the end of the $n^{\text {th }}$ occupation epoch of $x, y, z$ by the matter particle elapsed aft the $n^{\text {th }}$ occupation epoch.

$\bar{T}(x, y, z, n)$ is the average of the time periods:

$$
\begin{aligned}
& d t(x, y, z, 1)+\delta t(x, y, z, 1), d t(x, y, z, i)+\delta t(x, y, z, 2), \cdots \\
& \text { and } d t(x, y, z, n-1)+\delta t(x, y, z, n-1) .
\end{aligned}
$$

\section{Important note:}

1) The temporal variable $t(x, y, z, n)$ is representing a signal indexed by the occupation epoch number.

2) The term $\delta t(x, y, z, 0)+(n-1) \bar{T}(x, y, z, n)$ is representing a measure of the past temporal epoch before the $n^{\text {th }}$ occupation epoch of $x, y, z$ by the matter particle, the term $d t(x, y, z, n)$ is representing the a measure of the present $n^{\text {th }}$ occupation temporal epoch and the term $\delta t(x, y, z, n)$ is representing a measure of the future temporal epoch after the $n^{\text {th }}$ occupation epoch of $x, y, z$ by the matter particle. 


\subsection{Definition of the Infinitesimal Time as a Function of Infinitesimal Displacement of Space and Occupation Epoch Number}

In order to write infinitesimal time as a function of infinitesimal displacement of space and occupation epoch number suppose we have matter particle with rest mass $m_{0}$ move by speed $v$ with respect to some local observer through some space point $(x, y, z)$ at the $n^{\text {th }}$ occupation epoch of $(x, y, z)$ by the matter particle, then according to the special relativity theory the momentum of the particle is given by $p=\frac{m_{0} v}{\sqrt{1-\left(\frac{v}{c}\right)^{2}}}$, where $c$ is the speed of light in vacuum, now according of the wave-particle duality [6] if $\lambda(n)$ is representing the wavelength of this matter particle at the $n^{\text {th }}$ occupation epoch of $(x, y, z)$ by it then the momentum of this matter particle is also defined as following:

$$
p=\frac{h}{\lambda(n)}=\frac{m_{0} v}{\sqrt{1-\left(\frac{v}{c}\right)^{2}}}=\frac{m_{0}}{\sqrt{\frac{1}{v^{2}}-\frac{1}{c^{2}}}}
$$

where $h$ is the Planck's constant.

$$
\begin{gathered}
\rightarrow \sqrt{\frac{1}{v^{2}}-\frac{1}{c^{2}}}=\frac{m_{0} \lambda(n)}{h} \\
\rightarrow \frac{1}{v^{2}}-\frac{1}{c^{2}}=\frac{m_{0}^{2} \lambda(n)^{2}}{h^{2}} \\
\rightarrow \frac{1}{v^{2}}=\frac{1}{c^{2}}+\frac{m_{0}^{2} \lambda(n)^{2}}{h^{2}}=\frac{h^{2}+m_{0}^{2} \lambda(n)^{2} c^{2}}{h^{2} c^{2}} \\
\rightarrow v^{2}=\frac{\sum_{i=1}^{3} \sum_{j=1}^{3} g_{i j} d x^{i} * d x^{j}}{d t^{2}}=\frac{h^{2} c^{2}}{h^{2}+m_{0}^{2} \lambda(n)^{2} c^{2}} \\
\text { where }\left[\begin{array}{c}
d x^{1} \\
d x^{2} \\
d x^{3}
\end{array}\right]=\left[\begin{array}{l}
d x \\
d y \\
d z
\end{array}\right] \quad \text { is representing infinitesimal displacement vector of matter }
\end{gathered}
$$
particle at space point $(x, y, z), d t$ is representing infinitesimal displacement of time and $g_{i j}$ is the component of space metric tensor at the $i^{\text {th }}$ row and the $f^{\text {th }}$ column.

$$
\begin{gathered}
\rightarrow h^{2} c^{2} d t^{2}=\left(h^{2}+m_{0}^{2} \lambda(n)^{2} c^{2}\right) \sum_{i=1}^{3} \sum_{j=1}^{3} g_{i j} d x^{i} d x^{j} \\
\rightarrow d t=d t(d x, d y, d z, n)=\sqrt{\left(\frac{h^{2}+m_{0}^{2} \lambda(n)^{2} c^{2}}{h^{2} c^{2}}\right) \sum_{i=1}^{3} \sum_{j=1}^{3} g_{i j} d x^{i} d x^{j}}
\end{gathered}
$$

\section{Important notes:}

From the Equation (3) the speed of matter particle is defined by:

$$
v=\frac{h c}{\sqrt{h^{2}+m_{0}^{2} \lambda(n)^{2} c^{2}}}
$$


Thus speed of matter particle should always bounded by the speed of light in vacuum $c$ because:

$$
v=\frac{h c}{\sqrt{h^{2}+m_{0}^{2} \lambda(n)^{2} c^{2}}}<c
$$

For all matter particle possess non-zero mass $m_{0}>0$ and occupies non-zero volume of space $\lambda(n)>0$. Thus we can conclude that the speed of light in vacuum is representing with respect to the current theory is unsurpassable limit for all matter particles possess non-zero mass and occupies non-zero volume of space.

\subsection{The Real-Time State of Any Physical System Consisting of $N$ Matter Particles at Specified Space Point}

If we have a physical system consisting of $N$ matter particles $\left\{P_{1}, P_{2}, \cdots, P_{N}\right\}$ then the real-time state of this physical system at each space point $(x, y, z)$ is defined as following:

$$
\begin{aligned}
& \left|t\left(x, y, z, n_{1}, n_{2}, \cdots, n_{N}\right)\right\rangle=\left[\begin{array}{c}
t_{1}\left(x, y, z, n_{1}\right) \\
t_{2}\left(x, y, z, n_{2}\right) \\
\vdots \\
t_{N}\left(x, y, z, n_{N}\right)
\end{array}\right] \\
& t_{i}\left(x, y, z, n_{i}\right)= \begin{cases}\delta t_{i}(x, y, z, 0)+\left(n_{i}-1\right) \bar{T}_{i}\left(x, y, z, n_{i}\right)+d t_{i}\left(x, y, z, n_{i}\right)+\delta t_{i}\left(x, y, z, n_{i}\right), & n_{i} \in \mathbb{N} \\
0, & n_{i}=0\end{cases} \\
& \text { For all } i \in\{1,2, \cdots, N\} \text { : } \\
& \text { where: } n_{i} \text { is the occupation epoch number of the matter particle } P_{i} \text { at } \\
& (x, y, z) \text {. } \\
& \delta t_{i}(x, y, z, 0) \text { is the length of the initial leaving epoch elapsed before the first } \\
& \left|t\left(x, y, z, n_{1}, n_{2}, \cdots, n_{N}\right)\right\rangle=\left|P_{a}\left(x, y, z, n_{1}, n_{2}, \cdots, n_{N}\right)\right\rangle+\left|P_{r}\left(x, y, z, n_{1}, n_{2}, \cdots, n_{N}\right)\right\rangle \\
& +\left|F_{u}\left(x, y, z, n_{1}, n_{2}, \cdots, n_{N}\right)\right\rangle \\
& \left|P_{a}\left(x, y, z, n_{1}, n_{2}, \cdots, n_{N}\right)\right\rangle=\left[\begin{array}{c}
\delta t_{1}(x, y, z, 0)+\left(n_{1}-1\right) \bar{T}_{1}\left(x, y, z, n_{1}\right) \\
\delta t_{2}(x, y, z, 0)+\left(n_{2}-1\right) \bar{T}_{2}\left(x, y, z, n_{2}\right) \\
\vdots \\
\delta t_{N}(x, y, z, 0)+\left(n_{N}-1\right) \bar{T}_{N}\left(x, y, z, n_{N}\right)
\end{array}\right]
\end{aligned}
$$

\section{Important notes:}




$$
\begin{aligned}
\left|P_{r}\left(x, y, z, n_{1}, n_{2}, \cdots, n_{N}\right)\right\rangle & =\left[\begin{array}{c}
d t_{1}\left(x, y, z, n_{1}\right) \\
d t_{2}\left(x, y, z, n_{2}\right) \\
\vdots \\
d t_{N}\left(x, y, z, n_{N}\right)
\end{array}\right] \\
\left|F_{u}\left(x, y, z, n_{1}, n_{2}, \cdots, n_{N}\right)\right\rangle & =\left[\begin{array}{c}
\delta t_{1}\left(x, y, z, n_{1}\right) \\
\delta t_{2}\left(x, y, z, n_{2}\right) \\
\vdots \\
\delta t_{N}\left(x, y, z, n_{N}\right)
\end{array}\right]
\end{aligned}
$$

where: $\left|P_{a}\left(x, y, z, n_{1}, n_{2}, \cdots, n_{N}\right)\right\rangle,\left|P_{r}\left(x, y, z, n_{1}, n_{2}, \cdots, n_{N}\right)\right\rangle$ and $\left|F_{u}\left(x, y, z, n_{1}, n_{2}, \cdots, n_{N}\right)\right\rangle$ are called hereinafter past, present and future realtime state respectively.

\subsection{The Entanglement Translation of Real-Time State of Any} Physical System Consisting of $\boldsymbol{N}$ Matter Particles at Specified Space Point

If $\left|t\left(x, y, z, n_{1}, n_{2}, \cdots, n_{N}\right)\right\rangle=\left[\begin{array}{c}t_{1}\left(x, y, z, n_{1}\right) \\ t_{2}\left(x, y, z, n_{2}\right) \\ \vdots \\ t_{N}\left(x, y, z, n_{N}\right)\end{array}\right]$ is representing the real-time state of some physical system consisting of $N$ matter particles $\left\{P_{1}, P_{2}, \cdots, P_{N}\right\}$ at starting of the $n_{\mu}^{\text {th }}$ occupation of $(x, y, z)$ by the matter particle $P_{\mu}$ for some $\mu \in\{1,2, \cdots, N\}$ then the measurement process of the length of the $n_{\mu}^{\text {th }}$ occupation epoch of $(x, y, z)$ by the matter particle $P_{\mu}$ that result the $d t_{\mu}\left(x, y, z, n_{\mu}\right)$ is always transform the real-time state of physical system $\left|t\left(x, y, z, n_{1}, n_{2}, \cdots, n_{N}\right)\right\rangle=\left[\begin{array}{c}t_{1}\left(x, y, z, n_{1}\right) \\ t_{2}\left(x, y, z, n_{2}\right) \\ \vdots \\ t_{N}\left(x, y, z, n_{N}\right)\end{array}\right]$ according to the following Entanglement Translation:

$$
\begin{array}{r}
\left|t\left(x, y, z, n_{1}, n_{2}, \cdots, n_{N}\right)\right\rangle \rightarrow\left|t\left(x, y, z, n_{1}, n_{2}, \cdots, n_{N}\right)\right\rangle+|d t\rangle \\
|d t\rangle=d t_{\mu}\left(x, y, z, n_{\mu}\right)\left[\begin{array}{c}
t_{1}\left(x, y, z, n_{1}\right) *\left(t_{1}\left(x, y, z, n_{1}\right)\right)^{\nabla} \\
t_{2}\left(x, y, z, n_{2}\right) *\left(t_{2}\left(x, y, z, n_{2}\right)\right)^{\nabla} \\
\vdots \\
t_{N}\left(x, y, z, n_{N}\right) *\left(t_{N}\left(x, y, z, n_{N}\right)\right)^{\nabla}
\end{array}\right]
\end{array}
$$

For all $v \in\{1,2, \cdots, N\}$.

\section{Important note:}

1) if the $\left\{P_{1}, P_{2}, \cdots, P_{N}\right\}$ is representing a set of $N$ elementary matter particles then the occupation epoch is infinitesimal thus using the Equation (4) we can write the entanglement translation of $\left|t\left(x, y, z, n_{1}, n_{2}, \cdots, n_{N}\right)\right\rangle$ as following:

$$
\left|t\left(x, y, z, n_{1}, n_{2}, \cdots, n_{N}\right)\right\rangle \rightarrow\left|t\left(x, y, z, n_{1}, n_{2}, \cdots, n_{N}\right)\right\rangle+|d t\rangle
$$




$$
|d t\rangle=\sqrt{\left(\frac{h^{2}+m_{\mu}^{2} \lambda_{\mu}(n)^{2} c^{2}}{h^{2} c^{2}}\right) \sum_{i=1}^{3} \sum_{j=1}^{3} g_{i j} d x^{i} d x^{j}}\left[\begin{array}{c}
t_{1}\left(x, y, z, n_{1}\right) *\left(t_{1}\left(x, y, z, n_{1}\right)\right)^{\nabla} \\
t_{2}\left(x, y, z, n_{2}\right) *\left(t_{2}\left(x, y, z, n_{2}\right)\right)^{\nabla} \\
\vdots \\
\left.t_{N}\left(x, y, z, n_{N}\right) *\left(t_{N}\left(x, y, z, n_{N}\right)\right)\right)^{\nabla}
\end{array}\right]
$$

2) $\left(t_{v}\left(x, y, z, n_{v}\right)\right)^{\nabla}$ is representing the normalized reciprocal function of $t_{v}\left(x, y, z, n_{v}\right)$ which is normalized by removing the infinity from the range of reciprocal function $\frac{1}{t_{v}\left(x, y, z, n_{v}\right)}$.

3) $d t_{v}\left(x, y, z, n_{v}\right)=t_{v}\left(x, y, z, n_{v}\right) *\left(t_{v}\left(x, y, z, n_{v}\right)\right)^{\nabla} * d t_{\mu}\left(x, y, z, n_{\mu}\right)$ or equivalently: $\frac{d t_{n_{v}}^{v}\left(x, y, z, n_{v}\right)}{d t_{n_{\mu}}^{\mu}\left(x, y, z, n_{\mu}\right)}=t_{v}\left(x, y, z, n_{v}\right) *\left(t_{v}\left(x, y, z, n_{v}\right)\right)^{\nabla}=\left\{\begin{array}{ll}1, & n_{v} \in \mathbb{N} \\ 0, & n_{v}=0\end{array}\right.$ which is always result binary digits indicate wither the matter particle $P_{v}$ occupied $(x, y, z)$ or not and then determine whether the $v^{\text {th }}$ component of the Realtime state $\left|t\left(x, y, z, n_{1}, n_{2}, \cdots, n_{N}\right)\right\rangle$ is non-zero covariant under Entanglement Translation or remain zero contravariant, thus the Entanglement Translation is:

I. Pure covariant transformation when $n_{i}>0$ for all $i=1,2, \cdots, N$.

II. Pure contravariant transformation when $n_{i}=0$ for all $i=1,2, \cdots, N$.

III. Mixed covariant and contravariant transformation when $n_{i}>0$ and $n_{j}=0$ for some $i, j \in\{1,2, \cdots, N\}$.

4) according to this transformation the measurement process of the occupation of $(x, y, z)$ by the matter particle $P_{\mu}$ that result $d t_{\mu}\left(x, y, z, n_{\mu}\right)$ as observable quantity is the same to the measurement process of the part of leaving of $(x, y, z)$ by the rest matter particles of the physical system that are occupied and left $(x, y, z)$, thus the time of each one of these matter particle at of $(x, y, z)$-which is representing the total length of all occupation and leaving epochs of $(x, y, z)$ by the matter particle-should increase $d t_{\mu}\left(x, y, z, n_{\mu}\right)$ immediately at the end of measurement epoch, however if some matter particle of the physical system does not occupy $(x, y, z)$ from the starting of observation epoch until the starting $n_{\mu}^{\text {th }}$ occupation of $(x, y, z)$ by the matter particle $P_{\mu}$ then this matter particle will never occupy $(x, y, z)$ during the $n_{\mu}^{\text {th }}$ occupation epoch of $(x, y, z)$ by the matter particle $P_{\mu}$, and hence the time of this matter particle at $(x, y, z)$-which is representing of total length of all occupation and leaving epochs of $(x, y, z)$ by it-will never change from zero during the measurement epoch.

5) For all $\alpha, \beta \in\{1,2, \cdots, N\}$ :

$$
\begin{aligned}
D_{\beta}^{\alpha}\left(x, y, z, n_{1}, n_{2}, \cdots, n_{N}\right) & =d t_{\alpha}\left(x, y, z, n_{\alpha}\right) *\left(d t_{\beta}\left(x, y, z, n_{\beta}\right)\right)^{\nabla} \\
& =\left\{\begin{array}{l}
1, n_{\alpha} \neq 0 \text { and } n_{\beta} \neq 0 \\
0, \text { either } n_{\alpha}=0 \text { or } n_{\beta}=0
\end{array}\right.
\end{aligned}
$$

Which is representing tensor field that take the contravarinat vector $|d t\rangle$ 
and covariant vector

$$
\left\langle d t^{\nabla}\right|=\left(d t_{\mu}\left(x, y, z, n_{\mu}\right)\right)^{\nabla}\left[\begin{array}{c}
t_{1}\left(x, y, z, n_{1}\right) *\left(t_{1}\left(x, y, z, n_{1}\right)\right)^{\nabla} \\
t_{2}\left(x, y, z, n_{2}\right) *\left(t_{2}\left(x, y, z, n_{2}\right)\right)^{\nabla} \\
\vdots \\
t_{N}\left(x, y, z, n_{N}\right) *\left(t_{N}\left(x, y, z, n_{N}\right)\right)^{\nabla}
\end{array}\right]
$$

and produce $N^{2}$ components of the following matrix:

$$
D=D\left(\left\langle d t^{\nabla}|,| d t\right\rangle\right)=\left[\begin{array}{cccc}
d t_{1}\left(d t_{1}\right)^{\nabla} & d t_{1}\left(d t_{2}\right)^{\nabla} & \cdots & d t_{1}\left(d t_{N}\right)^{\nabla} \\
d t_{2}\left(d t_{1}\right)^{\nabla} & d t_{2}\left(d t_{2}\right)^{\nabla} & \cdots & d t_{2}\left(d t_{N}\right)^{\nabla} \\
\vdots & & \ddots & \\
d t_{N}\left(d t_{1}\right)^{\nabla} & d t_{N}\left(d t_{2}\right)^{\nabla} & \cdots & d t_{N}\left(d t_{N}\right)^{\nabla}
\end{array}\right]
$$

Such that $d t_{\alpha}=d t_{\alpha}\left(x, y, z, n_{\alpha}\right)$ and $\left(d t_{\beta}\right)^{\nabla}=\left(d t_{\beta}\left(x, y, z, n_{\beta}\right)\right)^{\nabla}$ thus:

$$
\begin{aligned}
D|d t\rangle & =\left[\begin{array}{cccc}
d t_{1}\left(d t_{1}\right)^{\nabla} & d t_{1}\left(d t_{2}\right)^{\nabla} & \cdots & d t_{1}\left(d t_{N}\right)^{\nabla} \\
d t_{2}\left(d t_{1}\right)^{\nabla} & d t_{2}\left(d t_{2}\right)^{\nabla} & \cdots & d t_{2}\left(d t_{N}\right)^{\nabla} \\
\vdots & & \ddots & \\
d t_{N}\left(d t_{1}\right)^{\nabla} & d t_{N}\left(d t_{2}\right)^{\nabla} & \cdots & d t_{N}\left(d t_{N}\right)^{\nabla}
\end{array}\right]\left[\begin{array}{c}
d t_{1} \\
d t_{2} \\
\vdots \\
d t_{N}
\end{array}\right] \\
& =\left\langle d t^{\nabla} \mid d t\right\rangle\left[\begin{array}{c}
d t_{1} \\
d t_{2} \\
\vdots \\
d t_{N}
\end{array}\right]=\left\langle d t^{\nabla} \mid d t\right\rangle|d t\rangle
\end{aligned}
$$

Such that: $\left\langle d t^{\nabla} \mid d t\right\rangle=d t_{1}\left(d t_{1}\right)^{\nabla}+d t_{2}\left(d t_{2}\right)^{\nabla}+\cdots+d t_{N}\left(d t_{N}\right)^{\nabla}$ is equivalent to the number of non-zero components of $|d t\rangle$ and the Real-time state $\left|t\left(x, y, z, n_{1}, n_{2}, \cdots, n_{N}\right)\right\rangle$.

Now:

$$
\begin{gathered}
D|d t\rangle=\left\langle d t^{\nabla} \mid d t\right\rangle|d t\rangle \\
\rightarrow D|d t\rangle-\left\langle d t^{\nabla} \mid d t\right\rangle|d t\rangle=|o\rangle
\end{gathered}
$$

such that $|o\rangle=\left[\begin{array}{c}0 \\ 0 \\ \vdots \\ 0\end{array}\right]$

$$
\rightarrow\left(D-\left\langle d t^{\nabla} \mid d t\right\rangle I_{N}\right)|d t\rangle=|o\rangle
$$

such that $I_{N}$ is $N \times N$ identity matrix

$$
\begin{aligned}
& \left(D-\left\langle d t^{\nabla} \mid d t\right\rangle I_{N}\right)\left(\left|t\left(x, y, z, n_{1}, n_{2}, \cdots, n_{N}\right)\right\rangle+|d t\rangle\right) \\
\rightarrow & =\left(D-\left\langle d t^{\nabla} \mid d t\right\rangle I_{N}\right)\left|t\left(x, y, z, n_{1}, n_{2}, \cdots, n_{N}\right)\right\rangle+\left(D-\left\langle d t^{\nabla} \mid d t\right\rangle I_{N}\right)|d t\rangle \\
= & \left(D-\left\langle d t^{\nabla} \mid d t\right\rangle I_{N}\right)\left|t\left(x, y, z, n_{1}, n_{2}, \cdots, n_{N}\right)\right\rangle
\end{aligned}
$$

Thus the Entanglement Translation: 
$\left|t\left(x, y, z, n_{1}, n_{2}, \cdots, n_{N}\right)\right\rangle \rightarrow\left|t\left(x, y, z, n_{1}, n_{2}, \cdots, n_{N}\right)\right\rangle+|d t\rangle$ is translational invariant with respect to the operator $\left(D-\left\langle d t^{\nabla} \mid d t\right\rangle I_{N}\right)$.

\subsection{The Real-Time Digital State of the $N$ Matter Particles Physical System at Specified Space Point}

If $\left|t\left(x, y, z, n_{1}, n_{2}, \cdots, n_{N}\right)\right\rangle=\left[\begin{array}{c}t_{1}\left(x, y, z, n_{1}\right) \\ t_{2}\left(x, y, z, n_{2}\right) \\ \vdots \\ t_{N}\left(x, y, z, n_{N}\right)\end{array}\right]$ is representing the real-time state of some physical system consisting of $N$ matter particles $\left\{P_{1}, P_{2}, \cdots, P_{N}\right\}$ at $(x, y, z)$ then the Real-time digital state at $(x, y, z)$ that is corresponding to $\left|t\left(x, y, z, n_{1}, n_{2}, \cdots, n_{N}\right)\right\rangle$ is defined as following:

$$
\left|\varphi\left(x, y, z, n_{1}, n_{2}, \cdots, n_{N}\right)\right\rangle=\left[\begin{array}{c}
\varphi_{1}\left(x, y, z, n_{1}\right) \\
\varphi_{2}\left(x, y, z, n_{2}\right) \\
\vdots \\
\varphi_{N}\left(x, y, z, n_{N}\right)
\end{array}\right]=\left[\begin{array}{c}
t_{1}\left(x, y, z, n_{1}\right) *\left(t_{1}\left(x, y, z, n_{1}\right)\right)^{\nabla} \\
t_{2}\left(x, y, z, n_{2}\right) *\left(t_{2}\left(x, y, z, n_{2}\right)\right)^{\nabla} \\
\vdots \\
t_{N}\left(x, y, z, n_{N}\right) *\left(t_{N}\left(x, y, z, n_{N}\right)\right)^{\nabla}
\end{array}\right]
$$

\section{Important note:}

1) The value of $\varphi_{i}\left(x, y, z, n_{i}\right)$ indicate wither the matter particle $P_{i}$ occupied the point $(x, y, z)$ or not with respect to observer or measurement instrument tracking its motion of $P_{i}$ through $(x, y, z)$.

2) If $\left|t\left(x, y, z, n_{1}, n_{2}, \cdots, n_{N}\right)\right\rangle=\left[\begin{array}{c}t_{1}\left(x, y, z, n_{1}\right) \\ t_{2}\left(x, y, z, n_{2}\right) \\ \vdots \\ t_{N}\left(x, y, z, n_{N}\right)\end{array}\right]$ is representing the real-time

state of the physical system at starting of the $n_{\mu}^{\text {th }}$ occupation of $(x, y, z)$ by the matter particle $P_{\mu}$ for some $\mu \in\{1,2, \cdots, N\}$ and $d t_{\mu}\left(x, y, z, n_{\mu}\right)$ is the result of the measurement process of the length of the $n_{\mu}^{\text {th }}$ occupation epoch of $(x, y, z)$ by the matter particle $P_{\mu}$ then the Entanglement Translation of $\left|t\left(x, y, z, n_{1}, n_{2}, \cdots, n_{N}\right)\right\rangle$ is given as following:

$$
\left|t\left(x, y, z, n_{1}, n_{2}, \cdots, n_{N}\right)\right\rangle \rightarrow\left|t\left(x, y, z, n_{1}, n_{2}, \cdots, n_{N}\right)\right\rangle+|d t\rangle
$$

Such that

$$
\begin{aligned}
|d t\rangle & =d t_{\mu}\left(x, y, z, n_{\mu}\right)\left[\begin{array}{c}
t_{1}\left(x, y, z, n_{1}\right) *\left(t_{1}\left(x, y, z, n_{1}\right)\right)^{\nabla} \\
t_{2}\left(x, y, z, n_{2}\right) *\left(t_{2}\left(x, y, z, n_{2}\right)\right)^{\nabla} \\
\vdots \\
t_{N}\left(x, y, z, n_{N}\right) *\left(t_{N}\left(x, y, z, n_{N}\right)\right)^{\nabla}
\end{array}\right] \\
& =d t_{\mu}\left(x, y, z, n_{\mu}\right)\left|\varphi\left(x, y, z, n_{1}, n_{2}, \cdots, n_{N}\right)\right\rangle
\end{aligned}
$$

Thus the Real-time digital state $\left|\varphi\left(x, y, z, n_{1}, n_{2}, \cdots, n_{N}\right)\right\rangle$ is representing the base state of Entanglement Translation of the real-time state $\left|t\left(x, y, z, n_{1}, n_{2}, \cdots, n_{N}\right)\right\rangle$. 
However

$\left|\varphi\left(x, y, z, n_{1}, n_{2}, \cdots, n_{N}\right)\right\rangle \in B^{N}=\left\{\left[\begin{array}{c}b_{1} \\ b_{2} \\ \vdots \\ b_{N}\end{array}\right] \mid b_{i} \in\{0,1\}\right.$ for all $\left.i=1,2, \cdots, N\right\}$ which is consisting of $2^{N} \quad N$-tuples of binary digits thus the Real-time digital state $\left|\varphi\left(x, y, z, n_{1}, n_{2}, \cdots, n_{N}\right)\right\rangle$ as well as Entanglement Translation $\left|t\left(x, y, z, n_{1}, n_{2}, \cdots, n_{N}\right)\right\rangle \rightarrow\left|t\left(x, y, z, n_{1}, n_{2}, \cdots, n_{N}\right)\right\rangle+|d t\rangle$ are quantifying the motion of the constituent matter particles of the physical system through $(x, y, z)$, this quantification allow these matter particles move exclusively at a finite sequential set of digital levels defined in the following section.

3) $\left|\varphi\left(x, y, z, n_{1}, n_{2}, \cdots, n_{N}\right)\right\rangle=\hat{D}_{|t\rangle}|d t\rangle$

where:

$$
|t\rangle=\left|t\left(x, y, z, n_{1}, n_{2}, \cdots, n_{N}\right)\right\rangle
$$

and:

$$
\hat{D}_{|t\rangle}=\left[\begin{array}{cccc}
\left(t_{1}\left(x, y, z, n_{1}\right)\right)^{\nabla} & 0 & \cdots & 0 \\
0 & \left(t_{2}\left(x, y, z, n_{2}\right)\right)^{\nabla} & \cdots & 0 \\
\vdots & & \ddots & \\
0 & 0 & \cdots & \left(t_{N}\left(x, y, z, n_{N}\right)\right)^{\nabla}
\end{array}\right]
$$

Thus we can write the Entanglement Translation of $\left|t\left(x, y, z, n_{1}, n_{2}, \cdots, n_{N}\right)\right\rangle$ :

$$
|t\rangle \rightarrow|t+d t\rangle=|t\rangle+|d t\rangle
$$

Such that

$$
|d t\rangle=d t_{\mu}\left(x, y, z, n_{\mu}\right)\left[\begin{array}{c}
t_{1}\left(x, y, z, n_{1}\right) *\left(t_{1}\left(x, y, z, n_{1}\right)\right)^{\nabla} \\
t_{2}\left(x, y, z, n_{2}\right) *\left(t_{2}\left(x, y, z, n_{2}\right)\right)^{\nabla} \\
\vdots \\
t_{N}\left(x, y, z, n_{N}\right) *\left(t_{N}\left(x, y, z, n_{N}\right)\right)^{\nabla}
\end{array}\right]
$$

As following:

$$
\begin{gathered}
|t\rangle \rightarrow|t+d t\rangle=|t\rangle+\frac{\delta}{\delta t_{\mu}}|t\rangle \\
\frac{\delta}{\delta t_{\mu}}=d t_{\mu}\left(x, y, z, n_{\mu}\right) \hat{D}_{|t\rangle} \\
\hat{D}_{|t\rangle}=\left[\begin{array}{cccc}
\left(t_{1}\left(x, y, z, n_{1}\right)\right)^{\nabla} & 0 & \cdots & 0 \\
0 & \left(t_{2}\left(x, y, z, n_{2}\right)\right)^{\nabla} & \cdots & 0 \\
\vdots & & \ddots & \\
0 & 0 & \cdots & \left(t_{N}\left(x, y, z, n_{N}\right)\right)^{\nabla}
\end{array}\right] \\
\hat{T}=d t_{\mu}\left(x, y, z, n_{\mu}\right) \hat{D}_{|t\rangle}+I_{N}
\end{gathered}
$$




\subsection{The Digital Levels of Real-Time Digital States of the Physical System Consisting of $N$ Matter Particles}

For any physical system $S$ consisting of $N$ matter particles $\left\{P_{1}, P_{2}, \cdots, P_{N}\right\}$ and for all $n=0,1,2, \cdots, N$ the digital level $n$ of $S$ is defined as the set of all possible real-time digital states of $S$ at any space point $(x, y, z)$ consisting of $n$ components equivalent to one and rest components equivalent to zero, thus if $\sigma_{N}(n)$ is representing the set of all subsets of $\{1,2, \cdots, N\}$ that are consisting of $n$ elements, and for any set $A$ and $B \delta_{B}^{A}=\left\{\begin{array}{l}1, B \subseteq A \\ 0, \text { otherwise }\end{array}\right.$ then the $n^{\text {th }}$ digital level of $S$ is defined as following:

$$
L_{N}(n)=\left\{\left[\begin{array}{c}
\delta_{\{1\}}^{s} \\
\delta_{\{2\}}^{s} \\
\vdots \\
\delta_{\{N\}}^{s}
\end{array}\right] \mid s \in \sigma_{N}(n)\right\}
$$

which their element are defined as surjective function $\left|\varphi_{n}\right\rangle: \sigma_{N}(n) \rightarrow L_{N}(n)$ such that for each $s \in \sigma_{N}(n)$ :

$$
\left|\varphi_{n}(s)\right\rangle=\left[\begin{array}{c}
\delta_{\{1\}}^{s} \\
\delta_{\{2\}}^{s} \\
\vdots \\
\delta_{\{N\}}^{s}
\end{array}\right]
$$

Important note:

1) If $\left|\varphi\left(x, y, z, n_{1}, n_{2}, \cdots, n_{N}\right)\right\rangle=\left[\begin{array}{c}t_{1}\left(x, y, z, n_{1}\right) *\left(t_{2}\left(x, y, z, n_{1}\right)\right)^{\nabla} \\ t_{2}\left(x, y, z, n_{2}\right) *\left(t_{2}\left(x, y, z, n_{2}\right)\right)^{\nabla} \\ \vdots \\ t_{N}\left(x, y, z, n_{N}\right) *\left(t_{N}\left(x, y, z, n_{N}\right)\right)^{\nabla}\end{array}\right]$ is representing the real-time digital state of some physical system consisting of $N$ matter particles $\left\{P_{1}, P_{2}, \cdots, P_{N}\right\}$ at $(x, y, z)$, then there exists:

$$
\begin{aligned}
n & =\left[\left(t_{1}\left(x, y, z, n_{1}\right)\right)^{\nabla}\left(t_{2}\left(x, y, z, n_{2}\right)\right)^{\nabla}\left(t_{N}\left(x, y, z, n_{N}\right)\right)^{\nabla}\right] \cdot\left[\begin{array}{c}
t_{1}\left(x, y, z, n_{1}\right) \\
t_{2}\left(x, y, z, n_{2}\right) \\
\vdots \\
t_{2}\left(x, y, z, n_{2}\right)
\end{array}\right] \\
& =\sum_{i=1}^{N}\left(t_{i}\left(x, y, z, n_{i}\right)\right)^{\nabla} * t_{i}\left(x, y, z, n_{i}\right)
\end{aligned}
$$

and $s=\bigcup_{t_{i}\left(x, y, z, n_{i}\right) \neq 0}^{N}(\{i\})$ fulfills:

$$
\left|\varphi\left(x, y, z, n_{1}, n_{2}, \cdots, n_{N}\right)\right\rangle=\left|\varphi_{n}(s)\right\rangle=\left[\begin{array}{c}
\delta_{\{1\}}^{s} \\
\delta_{\{2\}}^{s} \\
\vdots \\
\delta_{\{N\}}^{s}
\end{array}\right]
$$

2) If $\left|\varphi\left(x, y, z, n_{1}, n_{2}, \cdots, n_{N}\right)\right\rangle$ is real-time digital state of some physical system consisting of the matter particles $\left\{P_{1}, P_{2}, \cdots, P_{N}\right\}$ at $(x, y, z)$ then before the 
first occupation epochs of $(x, y, z)$ by any matter particle belong to $\left\{P_{1}, P_{2}, \cdots, P_{N}\right\}$-with respect to some observer or measurement instrument tracing the motion of the matter particles $\left\{P_{1}, P_{2}, \cdots, P_{N}\right\}$ through $(x, y, z)-$ the real-time digital state of the of the physical system at $(x, y, z)$ is equivalent to the equilibrium state

$\left|\varphi\left(x, y, z, n_{1}, n_{2}, \cdots, n_{N}\right)\right\rangle=|\varphi(x, y, z, 0,0, \cdots, 0)\rangle=\left|\varphi_{0}(\{\})\right\rangle=\left[\begin{array}{c}0 \\ 0 \\ \vdots \\ 0\end{array}\right]$ called hereinafter the falsehood digital state at $(x, y, z)$ which is representing the unique element of the digital level 0 of the system, then this state either stay in the digital level 0 along the observation epoch of the physical system or change due the occupation of $(x, y, z)$ by one matter particle belong to $\left\{P_{1}, P_{2}, \cdots, P_{N}\right\}$ to one state in the digital level 1 which is level consisting of all real-time digital states with one components equal one and rest components equal zero, then this state either stay in the digital level 1 or change due the occupation of $(x, y, z)$ by new one matter particle belong to $\left\{P_{1}, P_{2}, \cdots, P_{N}\right\}$ to one state in digital level 2 which is level consisting of all digital states with two components equal one and rest components equal zero, and so on until the digital state of the physical system reach the stationary equilibrium digital state $\left|\varphi\left(x, y, z, n_{1}, n_{2}, \cdots, n_{N}\right)\right\rangle=\left[\begin{array}{c}1 \\ 1 \\ \vdots \\ 1\end{array}\right]$ at the digital level $N$ and then resisting at this digital state for rest of observation epoch of the physical system. However we must keep in mind the impossibility of transition the Real-time digital state $\left|\varphi\left(x, y, z, n_{1}, n_{2}, \cdots, n_{N}\right)\right\rangle$ to another Real-time digital state belong to the same or lower digital level or to another Real-time digital state belong to higher digital level with $i^{\text {th }}$ component equivalent to zero for all $i \in\{1,2, \cdots, N\}$ fulfill $n_{i} \neq 0$ because each components of the digital state can only change from zero to one when some matter particle of the physical system start its first occupation epoch, Figure 1 representing an explanation of distribution of real-time digital states of any physical system consisting of 4 matter particles over their corresponding digital levels in addition to the possible transition of the Real-time digital states from different digital states to the Real-time digital states distributed in their near higher digital level.

3) If the constituent matter particles of the physical system $\left\{P_{1}, P_{2}, \cdots, P_{N}\right\}$ are distributed into set of finite orbits such as the distribution of electrons in atoms then for each one of these orbits the real-time digital state of the physical system at each space point belong to it will be the same when all matter particles at that orbit occupy all space points belong to it, however this symmetry of digital states at that orbit can break by the jumping of one matter particles to that orbit which can transit all points occupied by it at that orbit to the same digital state belong to next higher digital level, thus the distribution of the constituent 

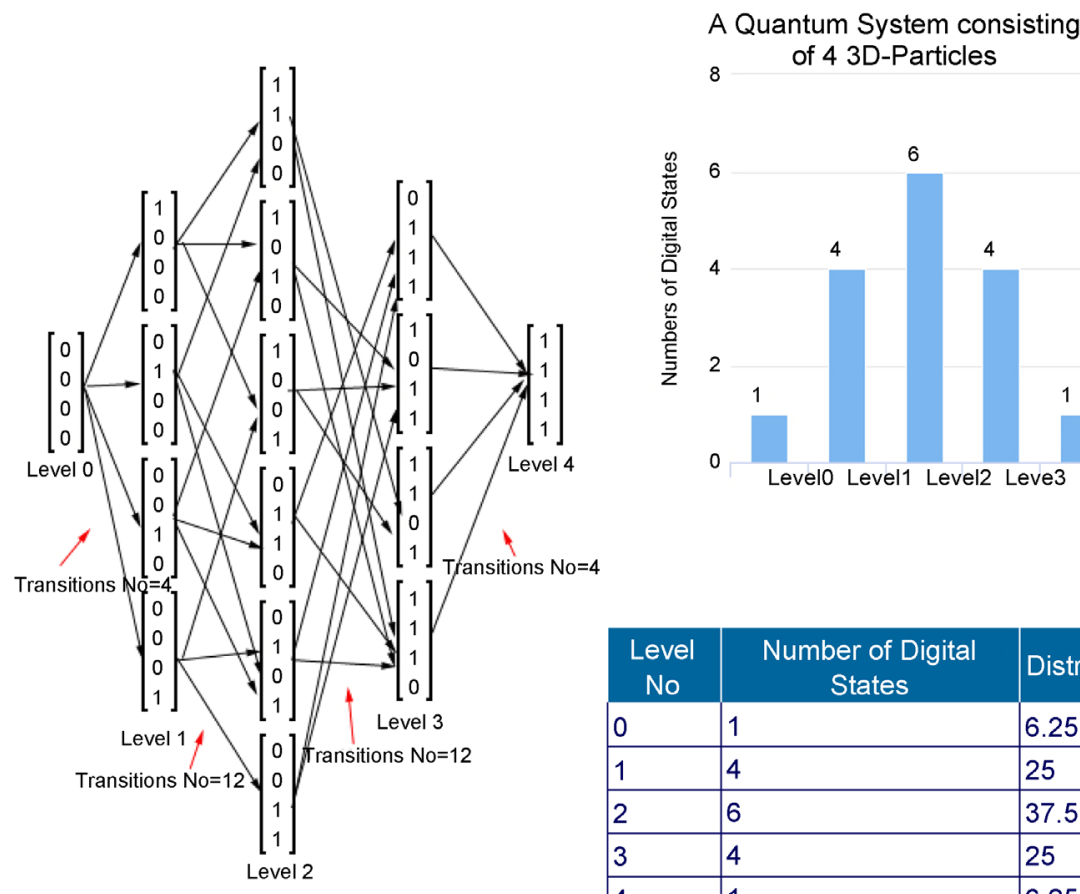

8 of 4 3D-Particles

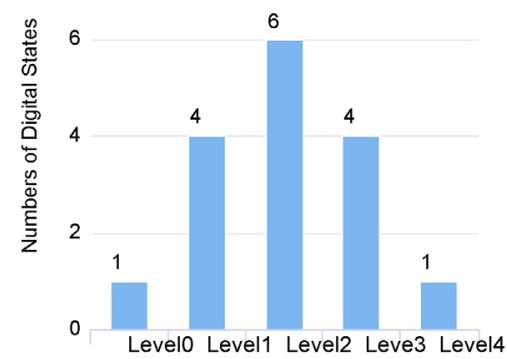

Figure 1. Illustration of digital levels and all possible transition between the real-time digital states belong to them for any physical system consisting of 4 matter particles.

matter particles into set of finite orbits is equivalent to the distribution of it into set of finite digital levels such that jumping of matter particle from initial orbit to the final orbit is equivalent to the transition of digital states at all points occupied by it at the final orbit to the same real-time digital state belong to next higher digital level.

\subsection{The Real-Time Transition State}

For any physical system $S$ consisting of $N$ matter particles $\left\{P_{1}, P_{2}, \cdots, P_{N}\right\}$ the real-time transition state of the physical system at $(x, y, z)$ that is corresponding to its real-time digital state at $(x, y, z)$ :

$$
\left|\varphi\left(x, y, z, n_{1}, n_{2}, \cdots, n_{N}\right)\right\rangle=\left|\varphi_{n}(s)\right\rangle=\left[\begin{array}{c}
\delta_{\{1\}}^{s} \\
\delta_{\{2\}}^{s} \\
\vdots \\
\delta_{\{N\}}^{s}
\end{array}\right] \text { is defined as the superposition of all }
$$

real-time digital states at $(x, y, z)$ that the physical system can transit to it at the of the next occupation epochs of $(x, y, z)$ by one of its constituent matter particles which is defined as following:

$$
\left|\psi_{n}(s)\right\rangle=\alpha_{s}\left|\varphi_{n}(s)\right\rangle+\sum_{\substack{u \in \sigma_{N}(n+1) \\ s \subset u}} \alpha_{u}\left|\varphi_{n+1}(u)\right\rangle
$$

Fulfills:

$$
\begin{gathered}
\hat{T}_{n}(s)\left|\psi_{n}(s)\right\rangle=\left|\varphi_{n}(s)\right\rangle \\
\hat{T}_{n+1}(u)\left|\psi_{n}(s)\right\rangle=\left|\varphi_{n+1}(u)\right\rangle
\end{gathered}
$$


For all $u \in \sigma_{N}(n+1)$ fulfills $s \subset u$.

Such that:

$$
\begin{aligned}
\hat{T}_{n}(s)=\left|\varphi_{n}(s)\right\rangle \otimes\left\langle\varphi_{n}(s)\right|= & {\left[\begin{array}{cccc}
\delta_{\{1\}}^{s}\left(\delta_{\{1\}}^{s}\right)^{\nabla} & \delta_{\{1\}}^{s}\left(\delta_{\{2\}}^{s}\right)^{\nabla} & \cdots & \delta_{\{1\}}^{s}\left(\delta_{\{N\}}^{s}\right)^{\nabla} \\
\delta_{\{2\}}^{s}\left(\delta_{\{1\}}^{s}\right)^{\nabla} & \delta_{\{2\}}^{s}\left(\delta_{\{2\}}^{s}\right)^{\nabla} & \cdots & \delta_{\{2\}}^{s}\left(\delta_{\{N\}}^{s}\right)^{\nabla} \\
\vdots & \vdots & \ddots & \vdots \\
\delta_{\{N\}}^{s}\left(\delta_{\{1\}}^{s}\right)^{\nabla} & \delta_{\{N\}}^{s}\left(\delta_{\{2\}}^{s}\right)^{\nabla} & \cdots & \delta_{\{N\}}^{s}\left(\delta_{\{N\}}^{s}\right)^{\nabla}
\end{array}\right](30) } \\
\hat{T}_{n+1}(u)=\left|\varphi_{n+1}(u)\right\rangle \otimes\left\langle\varphi_{n+1}(u)\right|= & {\left[\begin{array}{cccc}
\delta_{\{1\}}^{u}\left(\delta_{\{1\}}^{u}\right)^{\nabla} & \delta_{\{1\}}^{u}\left(\delta_{\{2\}}^{u}\right)^{\nabla} & \cdots & \delta_{\{1\}}^{u}\left(\delta_{\{N\}}^{u}\right)^{\nabla} \\
\delta_{\{2\}}^{u}\left(\delta_{\{1\}}^{u}\right)^{\nabla} & \delta_{\{2\}}^{u}\left(\delta_{\{2\}}^{u}\right)^{\nabla} & \cdots & \delta_{\{2\}}^{u}\left(\delta_{\{N\}}^{u}\right)^{\nabla} \\
\vdots & \vdots & \ddots & \vdots \\
\delta_{\{N\}}^{u}\left(\delta_{\{1\}}^{u}\right)^{\nabla} & \delta_{\{N\}}^{u}\left(\delta_{\{2\}}^{u}\right)^{\nabla} & \cdots & \delta_{\{N\}}^{u}\left(\delta_{\{N\}}^{u}\right)^{\nabla}
\end{array}\right](31) }
\end{aligned}
$$

$\alpha_{s} \in \mathbb{R}$ fulfills $\alpha_{\{\}}=0$ and $\alpha_{u} \in \mathbb{R}$ for all $u \in \sigma_{N}(n+1)$ fulfills $s \subset u$. where: $\otimes$ is the tensor product (outer product) operation.

$$
\left\langle\varphi_{n}(s)\right|=\left(\left|\varphi_{n}(s)\right\rangle\right)^{\nabla} \text { and }\left\langle\varphi_{n+1}(u)\right|=\left(\left|\varphi_{n+1}(u)\right\rangle\right)^{\nabla} \text { are normalized reciprocal }
$$
transpose of $\left|\varphi_{n}(s)\right\rangle$ and $\left|\varphi_{n+1}(u)\right\rangle$ respectively which are defined by taking the normalized reciprocal of the components of transpose of $\left|\varphi_{n}(s)\right\rangle$ and $\left|\varphi_{n+1}(u)\right\rangle$ respectively.

\section{Important note:}

1) When the physical system at the real-time digital state:

$$
\left|\varphi\left(x, y, z, n_{1}, n_{2}, \cdots, n_{N}\right)\right\rangle=\left|\varphi_{n}(s)\right\rangle=\left[\begin{array}{c}
\delta_{\{1\}}^{s} \\
\delta_{\{2\}}^{s} \\
\vdots \\
\delta_{\{N\}}^{s}
\end{array}\right] \text { the next occupation epochs of }
$$

$(x, y, z)$ by one of its constituent matter particles $P$ is either leaves the physical system at the real-time digital state $\left|\varphi_{n}(s)\right\rangle$ in a case that $P$ occupied $(x, y, z)$ at the previous occupation epoch or transit the real-time digital state to some state at the next higher digital level $\left|\varphi_{n+1}(u)\right\rangle$ such that $u \in \sigma_{N}(n+1)$ fulfills $s \subset u$, thus the real-time transition state is defined as superposition of its current real-time digital state and all real-time digital states at the next higher digital level that the physical system that can transit to them.

2) For all $i=1,2, \cdots, N$ the components of $\hat{T}_{n}(s)$ and $\hat{T}_{n+1}(u)$ at the $i^{\text {th }}$ row and the $f^{\text {th }}$ column which are $\delta_{\{i\}}^{s}\left(\delta_{\{j\}}^{s}\right)^{\nabla}=\delta_{\{i\}}^{s}\left(\delta_{\{j\}}^{s}\right)$ and $\delta_{\{i\}}^{u}\left(\delta_{\{j\}}^{u}\right)^{\nabla}=\delta_{\{i\}}^{u}\left(\delta_{\{j\}}^{u}\right)$ respectively indicate wither both the matter particles $P_{i}$ and $P_{j}$ occupied $(x, y, z)$ or not, thus $\hat{T}_{n}(s)$ and $\hat{T}_{n+1}(u)$ can play the role of observables operators that the Hermition matrices played in quantum mechanics because they are either leave the physical system at its current real-time digital state or transit it to one real-time digital state in next higher digital level when they act on the real-time transition state, however we use the normalized reciprocal as involution function instead of complex conjugate that used in quantum mechanics, so 
$\hat{T}_{n}(s)$ and $\hat{T}_{n+1}(u)$ are equal to their normalized reciprocal transposes in same way that the Hermition matrices equal to their complex conjugate transposes. In computation term $\hat{T}_{n}(s)$ and $\hat{T}_{n+1}(u)$ are representing irreversible gates that forward the time of each constituent particles of the physical system only at the direction of increasing the number of occupation and leaving epochs of space points by it.

3) we can calculate the coefficients $\alpha_{s}$ and $\left\{\alpha_{u} \mid u \in \sigma_{N}(n+1)\right.$ fulfills $\left.s \subset u\right\}$ as following:

$$
\begin{aligned}
\text { Since for all } \boldsymbol{x} & =\left[\begin{array}{c}
x_{1} \\
x_{2} \\
\vdots \\
x_{n}
\end{array}\right], \boldsymbol{y}=\left[\begin{array}{c}
y_{1} \\
y_{2} \\
\vdots \\
y_{n}
\end{array}\right] \text { and } \mathbf{z}=\left[\begin{array}{c}
z_{1} \\
z_{2} \\
\vdots \\
z_{n}
\end{array}\right]: \\
(\boldsymbol{x} \otimes \boldsymbol{y}) \mathbf{z} & =\left[\begin{array}{cccc}
x_{1} y_{1} & x_{1} y_{2} & \cdots & x_{1} y_{n} \\
x_{2} y_{1} & x_{2} y_{2} & \cdots & x_{2} y_{n} \\
\vdots & \vdots & \ddots & \vdots \\
x_{n} y_{1} & x_{n} y_{2} & \cdots & x_{n} y_{n}
\end{array}\right]\left[\begin{array}{c}
z_{1} \\
z_{2} \\
\vdots \\
z_{n}
\end{array}\right]=\left(\sum_{i=1}^{n} y_{i} z_{i}\right)\left[\begin{array}{c}
x_{1} \\
x_{2} \\
\vdots \\
x_{n}
\end{array}\right]=(\boldsymbol{y} \cdot \mathbf{z}) \boldsymbol{x}
\end{aligned}
$$

the conditions:

$$
\begin{gathered}
\hat{T}_{n}(s)\left|\psi_{n}(s)\right\rangle=\left|\varphi_{n}(s)\right\rangle \\
\hat{T}_{n+1}(u)\left|\psi_{n}(s)\right\rangle=\left|\varphi_{n+1}(u)\right\rangle
\end{gathered}
$$

Implied that:

$$
\begin{gathered}
\left(\left\langle\varphi_{n}(s)|\cdot| \psi_{n}(s)\right\rangle\right)\left|\varphi_{n}(s)\right\rangle=\left|\varphi_{n}(s)\right\rangle \\
\left(\left\langle\varphi_{n+1}(u)|\cdot| \psi_{n}(s)\right\rangle\right)\left|\varphi_{n+1}(u)\right\rangle=\left|\varphi_{n+1}(u)\right\rangle
\end{gathered}
$$

And then:

$$
\begin{gathered}
\left\langle\varphi_{n}(s)|\cdot| \psi_{n}(s)\right\rangle=1 \\
\left\langle\varphi_{n+1}(u)|\cdot| \psi_{n}(s)\right\rangle=1
\end{gathered}
$$

So if $u_{1}, u_{2}, \cdots, u_{k}$ are representing the elements of $\left\{\alpha_{u} \mid u \in \sigma_{N}(n+1)\right.$ fulfills $\left.s \subset u\right\}$ for some integer $k \in\{1,2, \cdots, N\}$ then:

$$
\left|\psi_{n}(s)\right\rangle=\alpha_{s}\left|\varphi_{n}(s)\right\rangle+\sum_{i=1}^{k} \alpha_{u_{i}}\left|\varphi_{n+1}\left(u_{i}\right)\right\rangle
$$

And:

$$
\begin{aligned}
\left\langle\varphi_{n}(s)|\cdot| \psi_{n}(s)\right\rangle= & \left(\left\langle\varphi_{n}(s)|\cdot| \varphi_{n}(s)\right\rangle\right) \alpha_{s}+\sum_{i=1}^{k}\left(\left\langle\varphi_{n}(s)|\cdot| \varphi_{n+1}\left(u_{i}\right)\right\rangle\right) \alpha_{u_{i}}=1 \\
\left\langle\varphi_{n+1}\left(u_{1}\right)|\cdot| \psi_{n}(s)\right\rangle= & \left(\left\langle\varphi_{n+1}\left(u_{1}\right)|\cdot| \varphi_{n}(s)\right\rangle\right) \alpha_{s}+\sum_{i=1}^{k}\left(\left\langle\varphi_{n+1}\left(u_{1}\right)|\cdot| \varphi_{n+1}\left(u_{i}\right)\right\rangle\right) \alpha_{u_{i}}=1 \\
\left\langle\varphi_{n+1}\left(u_{2}\right)|\cdot| \psi_{n}(s)\right\rangle= & =\left(\left\langle\varphi_{n+1}\left(u_{2}\right)|\cdot| \varphi_{n}(s)\right\rangle\right) \alpha_{s}+\sum_{i=1}^{k}\left(\left\langle\varphi_{n+1}\left(u_{2}\right)|\cdot| \varphi_{n+1}\left(u_{i}\right)\right\rangle\right) \alpha_{u_{i}}=1 \\
& \vdots \\
\left\langle\varphi_{n+1}\left(u_{k}\right)|\cdot| \psi_{n}(s)\right\rangle= & =\left(\left\langle\varphi_{n+1}\left(u_{k}\right)|\cdot| \varphi_{n}(s)\right\rangle\right) \alpha_{s}+\sum_{i=1}^{k}\left(\left\langle\varphi_{n+1}\left(u_{k}\right)|\cdot| \varphi_{n+1}\left(u_{i}\right)\right\rangle\right) \alpha_{u_{i}}=1
\end{aligned}
$$




$$
\begin{gathered}
n \alpha_{s}+\sum_{i=1}^{k} n \alpha_{u_{i}}=1 \\
n \alpha_{s}+(n+1) \alpha_{u_{1}}+\sum_{i=2}^{k} n \alpha_{u_{i}}=1 \\
\rightarrow n \alpha_{s}+(n+1) \alpha_{u_{2}}+\sum_{\substack{i=1 \\
i \neq 2}}^{k} n \alpha_{u_{i}}=1 \\
\vdots \\
n \alpha_{s}+(n+1) \alpha_{u_{k}}+\sum_{i=1}^{k-1} n \alpha_{u_{i}}=1
\end{gathered}
$$

Now there is two possible cases of above linear system depend on the value of $n$ defined as following:

A. if $n=0$ then the linear system is reduced to:

$$
\alpha_{u_{1}}=1, \alpha_{u_{2}}=1, \cdots, \alpha_{u_{k}}=1
$$

And then:

$$
\left|\psi_{0}(\{\})\right\rangle=\left|\varphi_{1}\left(u_{1}\right)\right\rangle+\left|\varphi_{1}\left(u_{2}\right)\right\rangle+\cdots+\left|\varphi_{1}\left(u_{k}\right)\right\rangle=\left|\varphi_{1}(\{1\})\right\rangle+\left|\varphi_{1}(\{2\})\right\rangle+\cdots+\left|\varphi_{1}(\{N\})\right\rangle=\left[\begin{array}{c}
1 \\
1 \\
\vdots \\
1
\end{array}\right] \text {. }
$$

B. If $n>0$ then above linear system is defined in matrix form as following:

$$
\left[\begin{array}{cccc}
n & n & \cdots & n \\
n & (n+1) & \cdots & n \\
\vdots & \vdots & \ddots & \vdots \\
n & n & \cdots & (n+1)
\end{array}\right]\left[\begin{array}{c}
\alpha_{s} \\
\alpha_{u_{1}} \\
\vdots \\
\alpha_{u_{k}}
\end{array}\right]=\left[\begin{array}{c}
1 \\
1 \\
\vdots \\
1
\end{array}\right]
$$

Thus using the Cramer's rule [3] for solving the linear system we find:

$$
\begin{aligned}
\alpha_{s} & =\frac{\left|\begin{array}{cccc}
1 & n & \cdots & n \\
1 & (n+1) & \cdots & n \\
\vdots & \vdots & \ddots & \vdots \\
1 & n & \cdots & (n+1)
\end{array}\right|}{\left|\begin{array}{cccc}
n & n & \cdots & n \\
n & (n+1) & \cdots & n \\
\vdots & \vdots & \ddots & \vdots \\
n & n & \cdots & (n+1)
\end{array}\right|}, \alpha_{u_{1}}=\frac{\left|\begin{array}{cccc}
n & 1 & \cdots & n \\
n & 1 & \cdots & n \\
\vdots & \vdots & \ddots & \vdots \\
n & 1 & \cdots & (n+1)
\end{array}\right|}{\left|\begin{array}{cccc}
n & n & \cdots & n \\
n & (n+1) & \cdots & n \\
\vdots & \vdots & \ddots & \vdots \\
n & n & \cdots & (n+1)
\end{array}\right|}, \cdots, \\
\alpha_{u_{k}} & =\frac{\left|\begin{array}{cccc}
n & n & \cdots & 1 \\
n & (n+1) & \cdots & 1 \\
\vdots & \vdots & \ddots & \vdots \\
n & n & \cdots & 1
\end{array}\right|}{\left|\begin{array}{cccc}
n \\
n \\
n & (n+1) & \cdots & n \\
\vdots & \vdots & \ddots & \vdots \\
n & n & \cdots & (n+1)
\end{array}\right|}
\end{aligned}
$$


And hence the real-time transition state is defined as following:

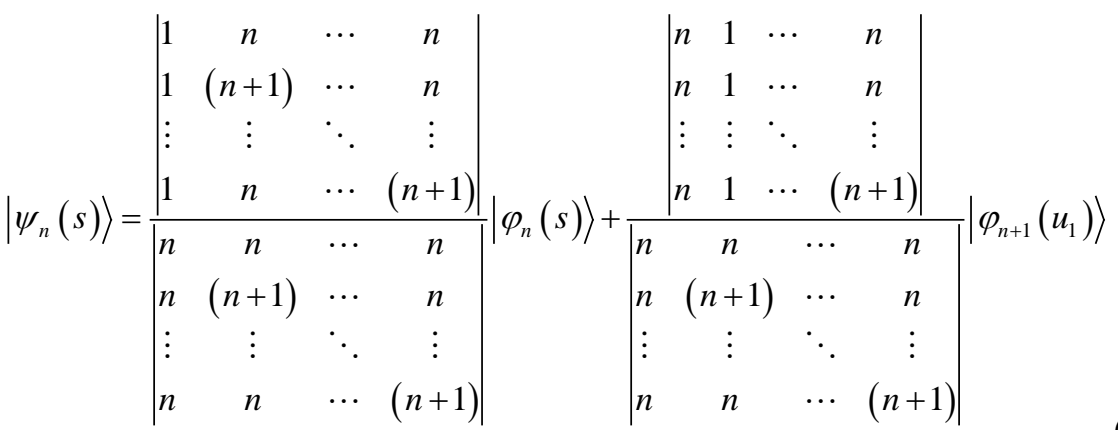

$$
\begin{aligned}
& +\cdots+\frac{\left|\begin{array}{cccc}
n & n & \cdots & 1 \\
n & (n+1) & \cdots & 1 \\
\vdots & \vdots & \ddots & \vdots \\
n & n & \cdots & 1
\end{array}\right|}{\left|\begin{array}{cccc}
n & n & \cdots & n \\
n & (n+1) & \cdots & n \\
\vdots & \vdots & \ddots & \vdots \\
n & n & \cdots & (n+1)
\end{array}\right|}\left|\varphi_{n+1}\left(u_{k}\right)\right\rangle
\end{aligned}
$$

\subsection{The Analog Occupies Path of the Physical System Consisting of $N$ Matter Particles}

The analog occupies path of $N$ matter particles physical system is the path that consisting of all real-time states at all space point occupied or occupies at least by one of its constituent matter particles.

\subsection{The Digital Occupies Path of the Physical System Consisting of $N$ Matter Particles}

The digital occupies path of $N$ matter particles physical system is the path that consisting of all real-time digital states at all space point occupied or occupies at least by one of its constituent matter particles.

\section{Mathematical Formulation: An Introduction to the Calculus of Fluctuation}

\subsection{The n-Dimensional Real Collinear Set}

For each $n \in \mathbb{N}$ the $\mathrm{n}$-dimensional real collinear set of any two point $\boldsymbol{p}, \boldsymbol{q} \in \mathbb{R}^{n}$ donated by $\mathbb{R}^{n}(\boldsymbol{p}, \boldsymbol{q})$ is defined as a set of all point in $\mathbb{R}^{n}$ lied at the line that contains $p$ and $q$.

\section{Example of real collinear set}

The set of real numbers is a 1-dimesional collinear set of any two real number $x$, y. i.e. $\mathbb{R}=\mathbb{R}^{1}(x, y), \forall x, y \in \mathbb{R}$.

\subsection{The n-Dimensional Displacement Vector}

For all $\boldsymbol{p}=\left(p_{1}, p_{2}, \cdots, p_{n}\right) \in \mathbb{R}^{n}$ and $\boldsymbol{q}=\left(q_{1}, q_{2}, \cdots, q_{n}\right) \in \mathbb{R}^{n}$ the n-dimensional displacement vector from $\boldsymbol{p}$ to $\boldsymbol{q}$ is defined as following: 


$$
\overrightarrow{\boldsymbol{d}}(\boldsymbol{p}, \boldsymbol{q})=\boldsymbol{q}-\boldsymbol{b}=\left[\begin{array}{c}
d_{1}(p, q) \\
d_{2}(p, q) \\
\vdots \\
d_{n}(p, q)
\end{array}\right]=\left[\begin{array}{c}
q_{1}-p_{1} \\
q_{2}-p_{2} \\
\vdots \\
q_{n}-p_{n}
\end{array}\right]
$$

\section{Important note:}

For all $\boldsymbol{p}=\left(p_{1}, p_{2}, \cdots, p_{n}\right) \in \mathbb{R}^{n}$ the author will donate to the vector $\overrightarrow{\boldsymbol{d}}(\boldsymbol{o}, \boldsymbol{p})=\left[\begin{array}{c}p_{1} \\ p_{2} \\ \vdots \\ p_{n}\end{array}\right]$ by $\overrightarrow{\boldsymbol{p}}$ such that $\boldsymbol{o}=(0,0, \cdots, 0) \in \mathbb{R}^{n}$.

\subsection{The Equilibrium Null Point and Vector}

The equilibrium null point is the point is the point $\boldsymbol{o}=(0,0, \cdots, 0) \in \mathbb{R}^{n}$ and the equilibrium null vector is vector $\overrightarrow{\boldsymbol{o}}=\left[\begin{array}{c}0 \\ 0 \\ \vdots \\ 0\end{array}\right]$.

\subsection{The Equilibrium Unity Point and Vector}

The equilibrium unity point is the point $\boldsymbol{u}=(1,1, \cdots, 1) \in \mathbb{R}^{n}$ and the equilibrium unity vector is the vector $\overrightarrow{\boldsymbol{d}}(\boldsymbol{o}, \boldsymbol{u})=\overrightarrow{\boldsymbol{u}}=\left[\begin{array}{c}1 \\ 1 \\ \vdots \\ 1\end{array}\right]$.

\subsection{Conditions of Positioning in n-Dimensional Collinear Set}

For each $n \in \mathbb{N}$ the $\mathrm{n}$-dimensional real collinear set of any two point $\boldsymbol{x}, \boldsymbol{y} \in \mathbb{R}^{n}$ such that $\boldsymbol{x}=\left(x_{1}, x_{2}, \cdots, x_{n}\right)$ and $\boldsymbol{y}=\left(y_{1}, y_{2}, \cdots, y_{n}\right)$ the conditions of positioning $\boldsymbol{q}=\left(q_{1}, q_{2}, \cdots, q_{n}\right) \in \mathbb{R}^{n}$ in $\mathbb{R}^{n}(\boldsymbol{x}, \boldsymbol{y})$ are defined as following:

1) The tangent and cotangent of the angle between the line that connect $\boldsymbol{x}$ and $\boldsymbol{q}$ should be equal to the tangent and cotangent of the angle between the line that connect $\boldsymbol{x}$ and $\boldsymbol{y}$, mathematically this condition is defined as following: $\frac{y_{j}-x_{j}}{y_{i}-x_{i}}=\frac{q_{j}-x_{j}}{q_{i}-x_{i}} \quad \forall i, j \in\{1,2, \cdots, n\} \quad$ satisfy $y_{i} \neq x_{i}$ and $y_{j} \neq x_{j}$.

2) The equivalent components of $\boldsymbol{x}$ and $\boldsymbol{y}$ should be equivalent to their corresponding components of $\boldsymbol{q}$, mathematically this condition is defined as following: $q_{k}=x_{k}=y_{k} \quad \forall k \in\{1,2, \cdots, n\}$ fulfills $x_{k}=y_{k}$.

\section{Important note:}

From the first condition $\forall i, j \in\{1,2, \cdots, n\}$ satisfy $y_{i} \neq x_{i}$ and $y_{j} \neq x_{j}$

$$
\begin{aligned}
q_{j} & =x_{j}+\frac{q_{i}-x_{i}}{y_{i}-x_{i}}\left(y_{j}-x_{j}\right)=x_{j}-\frac{q_{i}-x_{i}}{y_{i}-x_{i}} x_{j}+\frac{q_{i}-x_{i}}{y_{i}-x_{i}} y_{j} \\
& =\frac{y_{i}-q_{i}}{y_{i}-x_{i}} x_{j}+\frac{q_{i}-x_{i}}{y_{i}-x_{i}} y_{j}
\end{aligned}
$$


Also

$$
\begin{aligned}
q_{i} & =x_{i}+\frac{q_{j}-x_{j}}{y_{j}-x_{j}}\left(y_{i}-x_{i}\right)=x_{i}-\frac{q_{j}-x_{j}}{y_{j}-x_{j}} x_{i}+\frac{q_{j}-x_{j}}{y_{j}-x_{j}} y_{i} \\
& =\frac{y_{j}-q_{j}}{y_{j}-x_{j}} x i+\frac{q_{j}-x_{j}}{y_{j}-x_{j}} y_{i}
\end{aligned}
$$

From the second condition $\forall k \in\{1,2, \cdots, n\}$ satisfy $\quad x_{k}=y_{k}$ we find that

$$
\begin{aligned}
q_{k} & =\left(\frac{y_{j}-q_{j}}{y_{j}-x_{j}}+\frac{q_{j}-x_{j}}{y_{j}-x_{j}}\right) q_{k}=\frac{y_{j}-q_{j}}{y_{j}-x_{j}} q_{k}+\frac{q_{j}-x_{j}}{y_{j}-x_{j}} q_{k} \\
& =\frac{y_{j}-q_{j}}{y_{j}-x_{j}} x_{k}+\frac{q_{j}-x_{j}}{y_{j}-x_{j}} y_{k}
\end{aligned}
$$

Also

$$
\begin{gathered}
q_{k}=\left(\frac{y_{i}-q_{i}}{y_{i}-x_{i}}+\frac{q_{i}-x_{i}}{y_{i}-x_{i}}\right) q_{k}=\frac{y_{i}-q_{i}}{y_{i}-x_{i}} q_{k}+\frac{q_{i}-x_{i}}{y_{i}-x_{i}} q_{k} \\
=\frac{y_{i}-q_{i}}{y_{i}-x_{i}} x_{k}+\frac{q_{i}-x_{i}}{y_{i}-x_{i}} y_{k} \\
\rightarrow \boldsymbol{q}=\frac{y_{j}-q_{j}}{y_{j}-x_{j}} \boldsymbol{x}+\frac{q_{j}-x_{j}}{y_{j}-x_{j}} \boldsymbol{y}=\frac{y_{i}-q_{i}}{y_{i}-x_{i}} \boldsymbol{x}+\frac{q_{i}-x_{i}}{y_{i}-x_{i}} \boldsymbol{y} \\
\mathbb{R}^{n}(\boldsymbol{x}, \boldsymbol{y})=\left\{\boldsymbol{q}=\left(q_{1}, q_{2}, \cdots, q_{n}\right) \in \mathbb{R}^{n} \mid \boldsymbol{q}=\frac{y_{i}-q_{i}}{y_{i}-x_{i}} \boldsymbol{x}+\frac{q_{i}-x_{i}}{y_{i}-x_{i}} \boldsymbol{y}\right. \\
\left.\rightarrow \quad \text { for all } i \in\{1,2, \cdots, n\} \text { fulfills } y_{i} \neq x_{i}\right\}
\end{gathered}
$$

\subsection{The n-Dimensional Collinear Vectors Set}

If $\mathbb{R}^{n}(\boldsymbol{p}, \boldsymbol{q})$ is n-dimensional real collinear such that $\boldsymbol{p}, \boldsymbol{q} \in \mathbb{R}^{n}$ then the collinear vectors set of $\mathbb{R}^{n}(\boldsymbol{p}, \boldsymbol{q})$ donated by $\overrightarrow{\mathbb{R}^{n}}(\boldsymbol{p}, \boldsymbol{q})$ is the set of all displacement vectors in $\mathbb{R}^{n}$ that their heads are belonging to $\mathbb{R}^{n}(\boldsymbol{p}, \boldsymbol{q})$ and tails are equivalent to the equilibrium null point $\boldsymbol{o}=(0,0, \cdots, 0) \in \mathbb{R}^{n}$, which is defined as following:

$$
\overrightarrow{\mathbb{R}^{n}}(\boldsymbol{p}, \boldsymbol{q})=\left\{\overrightarrow{\boldsymbol{d}}(\boldsymbol{o}, \boldsymbol{x})=\overrightarrow{\boldsymbol{x}}=\boldsymbol{x}-\boldsymbol{o} \mid \boldsymbol{x} \in \mathbb{R}^{n}(\boldsymbol{p}, \boldsymbol{q})\right\}
$$

\section{Important note:}

For any $\boldsymbol{x}=\left(x_{1}, x_{2}, \cdots, x_{n}\right) \in \mathbb{R}^{n}(\boldsymbol{p}, \boldsymbol{q})$ the exists displacement vector $\overrightarrow{\boldsymbol{d}}(\boldsymbol{o}, \boldsymbol{x})=\overrightarrow{\boldsymbol{x}}$ with components equivalent to $x_{1}, x_{2}, \cdots, x_{n}$ thus for all $i \in\{1,2, \cdots, n\}$ fulfill $p_{i} \neq q_{i}: \quad \overrightarrow{\boldsymbol{x}}=\frac{q_{i}-x_{i}}{q_{i}-p_{i}} \overrightarrow{\boldsymbol{p}}+\frac{x_{i}-p_{i}}{q_{i}-p_{i}} \overrightarrow{\boldsymbol{q}}$ such that $\overrightarrow{\boldsymbol{p}}=\overrightarrow{\boldsymbol{d}}(\boldsymbol{o}, \boldsymbol{p})$ and $\overrightarrow{\boldsymbol{q}}=\overrightarrow{\boldsymbol{d}}(\boldsymbol{o}, \boldsymbol{q})$ because: $\quad \boldsymbol{x}=\frac{q_{i}-x_{i}}{q_{i}-p_{i}} \boldsymbol{p}+\frac{x_{i}-p_{i}}{q_{i}-p_{i}} \boldsymbol{q}$ and the components of $\boldsymbol{x}, \boldsymbol{p}$ and $\boldsymbol{q}$ are equivalent to the components of $\overrightarrow{\boldsymbol{x}}, \overrightarrow{\boldsymbol{p}}$ and $\overrightarrow{\boldsymbol{q}}$ respectively. $\nabla^{2} \phi=4 \pi G \rho$.

\subsection{Theorem (3.2)}

For all $\boldsymbol{x}=\left(x_{1}, x_{2}, \cdots, x_{n}\right) \in \mathbb{R}^{n} /\{\boldsymbol{o}\}$ the $\mathrm{n}$-dimensional displacement vector set 
$\overrightarrow{\mathbb{R}^{n}}(\boldsymbol{o}, \boldsymbol{x})$ is representing vector subspace of $\mathbb{R}^{n}$ and $\left(\overrightarrow{\mathbb{R}^{n}}(\boldsymbol{o}, \boldsymbol{x}),+\right)$ is representing subgroup of $\left(\mathbb{R}^{n},+\right)$.

Prove:

For all $\boldsymbol{x}=\left(x_{1}, x_{2}, \cdots, x_{n}\right) \in \mathbb{R}^{n}$, all $i \in\{1,2, \cdots, n\}$ fulfills $x_{i} \neq 0$, all $a, b, c \in \mathbb{R}$ and all $\overrightarrow{\boldsymbol{y}}, \overrightarrow{\mathbf{z}}, \overrightarrow{\boldsymbol{w}} \in \overrightarrow{\mathbb{R}^{n}}(\boldsymbol{o}, \boldsymbol{x})$ such that:

$$
\begin{gathered}
\overrightarrow{\boldsymbol{y}}=\left[\begin{array}{c}
y_{1} \\
y_{2} \\
\vdots \\
y_{n}
\end{array}\right]=\frac{x_{i}-y_{i}}{x_{i}-0} \overrightarrow{\boldsymbol{o}}+\frac{y_{i}-0}{x_{i}-0} \overrightarrow{\boldsymbol{x}}=\frac{y_{i}}{x_{i}} \overrightarrow{\boldsymbol{x}}, \\
\overrightarrow{\mathbf{z}}=\left[\begin{array}{c}
z_{1} \\
z_{2} \\
\vdots \\
z_{n}
\end{array}\right]=\frac{x_{i}-z_{i}}{x_{i}-0} \overrightarrow{\boldsymbol{o}}+\frac{z_{i}-0}{x_{i}-0} \overrightarrow{\boldsymbol{x}}=\frac{z_{i}}{x_{i}} \overrightarrow{\boldsymbol{x}}
\end{gathered}
$$

and

$$
\overrightarrow{\boldsymbol{w}}=\left[\begin{array}{c}
w_{1} \\
w_{2} \\
\vdots \\
w
\end{array}\right]=\frac{x_{i}-w_{i}}{x_{i}-0} \overrightarrow{\boldsymbol{o}}+\frac{w_{i}-0}{x_{i}-0} \overrightarrow{\boldsymbol{x}}=\frac{w_{i}}{x_{i}} \overrightarrow{\boldsymbol{x}}
$$

1) $\overrightarrow{\mathbb{R}^{n}}(\boldsymbol{o}, \boldsymbol{x}) \subset \mathbb{R}^{n}$.

2)

$$
\begin{aligned}
a \overrightarrow{\boldsymbol{y}}+b \overrightarrow{\mathbf{z}} & =\frac{a y_{i}}{x_{i}} \overrightarrow{\boldsymbol{x}}+\frac{b z_{i}}{x_{i}} \overrightarrow{\boldsymbol{x}}=\frac{a y_{i}+b z_{i}}{x_{i}} \overrightarrow{\boldsymbol{x}} \\
& =\left(\frac{x_{i}-a y_{i}+b z_{i}}{x_{i}-0} \overrightarrow{\boldsymbol{o}}+\frac{a y_{i}+b z_{i}-0}{x_{i}-0} \overrightarrow{\boldsymbol{x}}\right) \in \overrightarrow{\mathbb{R}^{n}}(\boldsymbol{o}, \boldsymbol{x}) .
\end{aligned}
$$

(Closure under ad-

dition and scalar multiplication).

3)

$$
\begin{aligned}
(\overrightarrow{\boldsymbol{y}}+\overrightarrow{\mathbf{z}})+\overrightarrow{\boldsymbol{w}} & =(\overrightarrow{\boldsymbol{y}}+\overrightarrow{\boldsymbol{z}}+\overrightarrow{\boldsymbol{w}})=\frac{y_{i}}{x_{i}} \overrightarrow{\boldsymbol{x}}+\frac{z_{i}}{x_{i}} \overrightarrow{\boldsymbol{x}}+\frac{w_{i}}{x_{i}} \overrightarrow{\boldsymbol{x}} \quad \text { (Associativity of addition). } \\
& =\left(\frac{y_{i}+z_{i}+w_{i}}{x_{i}} \overrightarrow{\boldsymbol{x}}\right) \in \overrightarrow{\mathbb{R}^{n}}(\boldsymbol{o}, \boldsymbol{x}) .
\end{aligned}
$$

4) $\overrightarrow{\boldsymbol{y}}+\overrightarrow{\boldsymbol{z}}=\overrightarrow{\boldsymbol{z}}+\overrightarrow{\boldsymbol{y}}=\frac{y_{i}}{x_{i}} \overrightarrow{\boldsymbol{x}}+\frac{z_{i}}{x_{i}} \overrightarrow{\boldsymbol{x}}=\left(\frac{y_{i}+z_{i}}{x_{i}} \overrightarrow{\boldsymbol{x}}\right) \in \overrightarrow{\mathbb{R}^{n}}(\boldsymbol{o}, \boldsymbol{x})$. (Commutatively of addition).

5) $\overrightarrow{\boldsymbol{y}}+\overrightarrow{\boldsymbol{o}}=\overrightarrow{\boldsymbol{o}}+\overrightarrow{\boldsymbol{y}}=\overrightarrow{\boldsymbol{y}}$. (Identity element of addition).

6) $-\overrightarrow{\boldsymbol{y}}=\frac{-y_{i}}{x_{i}} \overrightarrow{\boldsymbol{x}}=\left(\frac{x_{i}+y_{i}}{x_{i}-0} \overrightarrow{\boldsymbol{o}}+\frac{-y_{i}-0}{x_{i}-0} \overrightarrow{\boldsymbol{x}}\right) \in \overrightarrow{\mathbb{R}^{n}}(\boldsymbol{o}, \boldsymbol{x})$ fulfills $\overrightarrow{\boldsymbol{y}}+(-\overrightarrow{\boldsymbol{y}})=\frac{y_{i}-y_{i}}{x_{i}} \overrightarrow{\boldsymbol{x}}=\overrightarrow{\boldsymbol{o}} . \quad$ (Inverse element of addition).

7) $a(b \overrightarrow{\boldsymbol{y}})=(a b) \overrightarrow{\boldsymbol{y}}=\left[\begin{array}{c}a b y_{1} \\ a b y_{2} \\ \vdots \\ a b y_{n}\end{array}\right]$ (Compatibility of scalar multiplication with field multiplication). 
8) $a(\overrightarrow{\boldsymbol{y}}+\overrightarrow{\boldsymbol{z}})=a \overrightarrow{\boldsymbol{y}}+a \overrightarrow{\boldsymbol{z}} \quad$ (Distributivity of scalar multiplication with respect to vector addition).

9) $(a+b) \overrightarrow{\boldsymbol{y}}=a \overrightarrow{\boldsymbol{y}}+b \overrightarrow{\boldsymbol{y}} \quad$ (Distributivity of scalar multiplication with respect to field addition).

10) $1 * \vec{y}=\vec{y}$ (Identity element of scalar multiplication).

\subsection{The n-Dimensional Real State Space}

For all $\boldsymbol{x}=\left(x_{1}, x_{2}, \cdots, x_{n}\right) \in \mathbb{R}^{n} /\{\boldsymbol{o}\}$ such that $\boldsymbol{o}=(0,0, \cdots, 0) \in \mathbb{R}^{n}$ the n-dimensional real state space at $x$ is the vector subspace of $\mathbb{R}^{n}$ defined by $\left(\overrightarrow{\mathbb{R}^{n}}(\boldsymbol{o}, \boldsymbol{x}),\langle.,\rangle.\right)$ and endowed with inner product

$\langle.,\rangle:. \overrightarrow{\mathbb{R}^{n}}(\boldsymbol{o}, \boldsymbol{x}) \times \overrightarrow{\mathbb{R}^{n}}(\boldsymbol{o}, \boldsymbol{x}) \rightarrow \mathbb{R}$ such that for all $\overrightarrow{\boldsymbol{a}}=\left[\begin{array}{c}a_{1} \\ a_{2} \\ \vdots \\ a_{n}\end{array}\right] \in \overrightarrow{\mathbb{R}^{n}}(\boldsymbol{o}, \boldsymbol{x})$ and $\overrightarrow{\boldsymbol{b}}=\left[\begin{array}{c}b_{1} \\ b_{2} \\ \vdots \\ b_{n}\end{array}\right] \in \overrightarrow{\mathbb{R}^{n}}(\boldsymbol{o}, \boldsymbol{x})$

$$
\langle\overrightarrow{\boldsymbol{a}}, \overrightarrow{\boldsymbol{b}}\rangle=\overrightarrow{\boldsymbol{a}} \cdot \overrightarrow{\boldsymbol{b}}=(\overrightarrow{\boldsymbol{a}})^{\mathrm{T}} \overrightarrow{\boldsymbol{b}}=\sum_{i=1}^{n} a_{i} * b_{i} .
$$

\section{Important note:}

$\left(\overrightarrow{\mathbb{R}^{n}}(\boldsymbol{o}, \boldsymbol{x}),\langle.,\rangle.\right)$ is representing vector subspace of n-dimensional Euclidean space $\left(\boldsymbol{E}_{n},\langle.,\rangle.\right)=\left(\mathbb{R}^{n},\langle. .\rangle,\right)$ thus is representing inner product Hilbert space.

\subsection{Equilibrium and Non-Equilibrium Classification of n-Dimensional Real State Space}

For all $\boldsymbol{x}=\left(x_{1}, x_{2}, \cdots, x_{n}\right) \in \mathbb{R}^{n} /\{\boldsymbol{o}\}$ we can classify the $\mathrm{n}$-dimensional real state space $\left(\overrightarrow{\mathbb{R}^{n}}(\boldsymbol{o}, \boldsymbol{x}),\langle.,\rangle.\right)$ as:

1) Equilibrium $\mathrm{n}$-dimensional real state space in a case of $x_{\mu}=x_{v}$ for all $\mu, v \in\{1,2, \cdots, n\}$.

2) Non-Equilibrium $\mathrm{n}$-dimensional real state space in a case of $x_{\mu} \neq x_{v}$ for some $\mu, v \in\{1,2, \cdots, n\}$.

Important notes:

If $\left(\overrightarrow{\mathbb{R}^{n}}(\boldsymbol{o}, \boldsymbol{x}),\langle. .\rangle.\right)$ is equilibrium $\mathrm{n}$-dimensional real state space then: $\left(\overrightarrow{\mathbb{R}^{n}}(\boldsymbol{o}, \boldsymbol{x}),\langle.,\rangle.\right)=\left(\overrightarrow{\mathbb{R}^{n}}(\boldsymbol{o}, \boldsymbol{u}),\langle.,\rangle.\right)$.

\subsection{The Normalized Reciprocal of Real Scalar}

For any $x \in \mathbb{R}$ the normalized reciprocal of $x$ is defined as following:

$$
x^{\nabla}= \begin{cases}\frac{1}{x}, & x \neq 0 \\ 0, & x=0\end{cases}
$$

Important note: 
1) is called normalized reciprocal because the usual reciprocal of $x$ which is equal $\frac{1}{x}$ is contain infinity in his range when $x=0$, so it is normalized by removing this infinity from its range:

2) $\varphi(x)=x * x^{\nabla} \in\{0,1\}$ is representing binary digit.

3) The Dirac's delta function is related to normalized reciprocal as following:

$$
\delta(x)=1-\varphi(x)
$$

Which is equal zero at all $x \neq 0$ and one at $x=0$.

\subsection{The Normalized Reciprocal Transpose of the Matrix}

For any matrix $\boldsymbol{A}=\left[\begin{array}{cccc}a_{11} & a_{12} & \cdots & a_{1 n} \\ a_{21} & a_{22} & \cdots & a_{2 n} \\ \vdots & \vdots & \ddots & \vdots \\ a_{m 1} & a_{m 2} & \cdots & a_{m n}\end{array}\right] \in \mathbb{R}^{m \times n}$ the normalized reciprocal of

$\boldsymbol{A}$ is defined as following:

$$
\boldsymbol{A}^{\nabla}=\left[\begin{array}{cccc}
a_{11}{ }^{\nabla} & a_{21}{ }^{\nabla} & \cdots & a_{m 1}{ }^{\nabla} \\
a_{12}{ }^{\nabla} & a_{22}{ }^{\nabla} & \cdots & a_{m 2}{ }^{\nabla} \\
\vdots & \vdots & \ddots & \vdots \\
a_{1 n}{ }^{\nabla} & a_{2 n}{ }^{\nabla} & \cdots & a_{m n}{ }^{\nabla}
\end{array}\right] \in \mathbb{R}^{n \times m}
$$

\subsection{Signal Tensor Field}

At any point $\boldsymbol{x}=\left(x_{1}, x_{2}, \cdots, x_{n}\right) \in \mathbb{R}^{n}$ the signal tensor field is second order tensor that take $\overrightarrow{\boldsymbol{x}}$ from $\overrightarrow{\mathbb{R}^{n}}(\boldsymbol{o}, \boldsymbol{x})$ and its corresponding dual vector $\overrightarrow{\boldsymbol{x}}^{\nabla}$ from $\overrightarrow{\mathbb{R}^{n}}\left(\boldsymbol{o}, \boldsymbol{x}^{\nabla}\right)$ such that $\boldsymbol{x}^{\nabla}=\left(x_{1}^{\nabla}, x_{2}^{\nabla}, \cdots, x_{n}^{\nabla}\right)$ and produce $n^{2}$ components defined as following:

$$
\boldsymbol{T}\left(\overrightarrow{\boldsymbol{x}}, \overrightarrow{\boldsymbol{x}}^{\nabla}\right)=\overrightarrow{\boldsymbol{x}} \otimes \overrightarrow{\boldsymbol{x}}^{\nabla}=\left[\begin{array}{cccc}
x_{1} x_{1}^{\nabla} & x_{1} x_{2}^{\nabla} & \cdots & x_{1} x_{n}^{\nabla} \\
x_{2} x_{1}^{\nabla} & x_{2} x_{2}^{\nabla} & \cdots & x_{2} x_{n}^{\nabla} \\
\vdots & \vdots & \ddots & \vdots \\
x_{n} x_{1}^{\nabla} & x_{n} x_{2}^{\nabla} & \cdots & x_{n} x_{n}^{\nabla}
\end{array}\right]
$$

Or in tensor notation:

$$
\boldsymbol{T}_{v}^{\mu}=\boldsymbol{T}_{v}^{\mu}\left(\overrightarrow{\boldsymbol{x}}, \overrightarrow{\boldsymbol{x}}^{\nabla}\right)=x_{\mu} x_{v}^{\nabla}
$$

For all $\mu, v \in\{1,2, \cdots, n\}$.

\section{Important notes:}

1) For all $\mu \in\{1,2, \cdots, n\}$ the $\mu^{\text {th }}$ diagonal component of $\boldsymbol{T}_{v}^{\mu}$ which is equal $x_{\mu} x_{\mu}{ }^{\nabla}$ is corresponding to the $\mu^{\text {th }}$ components of the binary digital state binary state $b=\hat{D}_{x} \overrightarrow{\boldsymbol{x}}$ so the diagonal components of $\boldsymbol{T}_{v}^{\mu}$ are representing the digital components of it, and all the rest components of $\boldsymbol{T}_{v}^{\mu}$ for all $\mu \neq v$ are representing the analog normalized ratio between the components of $\vec{x}$ at different indices.

2) For all $i \in\{1,2, \cdots, n\}$ if $x_{i}=0$ then $x_{i} x_{i}^{\nabla}=x_{i} x_{j}^{\nabla}=x_{j} x_{i}^{\nabla}=0$ for all $j \in\{1,2, \cdots, n\}$ so in the signal tensor field the present and absent of the digital 
signal is restricted by the present and absent of its corresponding analog signals and vice versa.

3) $\boldsymbol{T}\left(\overrightarrow{\boldsymbol{y}}, \overrightarrow{\boldsymbol{y}}^{\nabla}\right)=\boldsymbol{T}\left(\overrightarrow{\boldsymbol{x}}, \overrightarrow{\boldsymbol{x}}^{\nabla}\right)$ at all point $\boldsymbol{y}=\left(y_{1}, y_{2}, \cdots, y_{n}\right) \in \mathbb{R}^{n}(\boldsymbol{o}, \boldsymbol{x}) /\{\boldsymbol{o}\}$ because for all $i \in\{1,2, \cdots, n\}$ fulfill $x_{i} \neq 0$ and $y_{i} \neq 0$

$$
\boldsymbol{y}=\frac{x_{i}-y_{i}}{x_{i}-0} \boldsymbol{o}+\frac{y_{i}-0}{x_{i}-0} \boldsymbol{x}=\frac{y_{i}}{x_{i}} \boldsymbol{x}
$$

thus:

$$
\begin{aligned}
& \boldsymbol{T}\left(\overrightarrow{\boldsymbol{y}}, \overrightarrow{\boldsymbol{y}}^{\nabla}\right)=\boldsymbol{T}\left(\frac{y_{i}}{x_{i}} \overrightarrow{\boldsymbol{x}},\left(\frac{y_{i}}{x_{i}} \overrightarrow{\boldsymbol{x}}\right)^{\nabla}\right)=\left(\frac{y_{i}}{x_{i}} \boldsymbol{x}\right) \otimes\left(\frac{y_{i}}{x_{i}} \boldsymbol{x}\right)^{\nabla} \\
& =\left[\begin{array}{cccc}
\frac{y_{i}}{x_{i}} x_{1}\left(\frac{y_{i}}{x_{i}} x_{1}\right)^{\nabla} & \frac{y_{i}}{x_{i}} x_{1}\left(\frac{y_{i}}{x_{i}} x_{2}\right)^{\nabla} & \cdots & \frac{y_{i}}{x_{i}} x_{1}\left(\frac{y_{i}}{x_{i}} x_{n}\right)^{\nabla} \\
\frac{y_{i}}{x_{i}} x_{2}\left(\frac{y_{i}}{x_{i}} x_{2}\right)^{\nabla} & \frac{y_{i}}{x_{i}} x_{2}\left(\frac{y_{i}}{x_{i}} x_{2}\right)^{\nabla} & \cdots & \frac{y_{i}}{x_{i}} x_{2}\left(\frac{y_{i}}{x_{i}} x_{n}\right)^{\nabla} \\
\vdots & \vdots & \ddots & \vdots \\
\frac{y_{i}}{x_{i}} x_{n}\left(\frac{y_{i}}{x_{i}} x_{n}\right)^{\nabla} & \frac{y_{i}}{x_{i}} x_{n}\left(\frac{y_{i}}{x_{i}} x_{2}\right)^{\nabla} & \cdots & \frac{y_{i}}{x_{i}} x_{n}\left(\frac{y_{i}}{x_{i}} x_{n}\right)^{\nabla}
\end{array}\right] \\
& =\left[\begin{array}{cccc}
x_{1} x_{1}^{\nabla} & x_{1} x_{2}^{\nabla} & \cdots & x_{1} x_{n}^{\nabla} \\
x_{2} x_{1}^{\nabla} & x_{2} x_{2}^{\nabla} & \cdots & x_{2} x_{n}^{\nabla} \\
\vdots & \vdots & \ddots & \vdots \\
x_{n} x_{1}^{\nabla} & x_{n} x_{2}^{\nabla} & \cdots & x_{n} x_{n}^{\nabla}
\end{array}\right]=\overrightarrow{\boldsymbol{x}} \otimes \overrightarrow{\boldsymbol{x}}^{\nabla}=\boldsymbol{T}\left(\overrightarrow{\boldsymbol{x}}, \overrightarrow{\boldsymbol{x}}^{\nabla}\right) \\
& \left(\boldsymbol{T}\left(\overrightarrow{\boldsymbol{x}}, \overrightarrow{\boldsymbol{x}}^{\nabla}\right)\right)^{\nabla}=\left[\begin{array}{cccc}
\left(x_{1} x_{1}^{\nabla}\right)^{\nabla} & \left(x_{2} x_{1}^{\nabla}\right)^{\nabla} & \cdots & \left(x_{n} x_{1}^{\nabla}\right)^{\nabla} \\
\left(x_{1} x_{2}^{\nabla}\right)^{\nabla} & \left(x_{2} x_{2}^{\nabla}\right)^{\nabla} & \cdots & \left(x_{n} x_{2}^{\nabla}\right)^{\nabla} \\
\vdots & \vdots & \ddots & \vdots \\
\left(x_{1} x_{n}^{\nabla}\right)^{\nabla} & \left(x_{2} x_{n}^{\nabla}\right)^{\nabla} & \cdots & \left(x_{n} x_{n}^{\nabla}\right)^{\nabla}
\end{array}\right] \\
& =\left[\begin{array}{cccc}
x_{1} x_{1}^{\nabla} & x_{1} x_{2}^{\nabla} & \cdots & x_{1} x_{n}^{\nabla} \\
x_{2} x_{1}^{\nabla} & x_{2} x_{2}^{\nabla} & \cdots & x_{2} x_{n}^{\nabla} \\
\vdots & \vdots & \ddots & \vdots \\
x_{n} x_{1}^{\nabla} & x_{n} x_{2}^{\nabla} & \cdots & x_{n} x_{n}^{\nabla}
\end{array}\right] \\
& \rightarrow\left(T\left(\vec{x}, \vec{x}^{\nabla}\right)\right)^{\nabla}=T\left(\vec{x}, \vec{x}^{\nabla}\right)
\end{aligned}
$$

or in tensor notation:

$$
\left(\boldsymbol{T}_{v}^{\mu}\right)^{\nabla}=\boldsymbol{T}_{v}^{\mu}
$$

For this reason $\boldsymbol{T}\left(\overrightarrow{\boldsymbol{x}}, \overrightarrow{\boldsymbol{x}}^{\nabla}\right)$ will play in the digital matter particle Physics the similar role that Herniation matrix or in general adjoin operator plays in quantum physics.

5) For all $\boldsymbol{x}=\left(x_{1}, x_{2}, \cdots, x_{n}\right) \in \mathbb{R}^{n}$ the vector $\overrightarrow{\boldsymbol{x}}=\left[\begin{array}{c}x_{1} \\ x_{2} \\ \vdots \\ x_{n}\end{array}\right]$ : 


$$
\begin{aligned}
\boldsymbol{T}\left(\overrightarrow{\boldsymbol{x}}, \overrightarrow{\boldsymbol{x}}^{\nabla}\right) \overrightarrow{\boldsymbol{x}} & =\left[\begin{array}{cccc}
x_{1} x_{1}^{\nabla} & x_{1} x_{2}{ }^{\nabla} & \cdots & x_{1} x_{n}{ }^{\nabla} \\
x_{2} x_{1}^{\nabla} & x_{2} x_{2}{ }^{\nabla} & \cdots & x_{2} x_{n}^{\nabla} \\
\vdots & \vdots & \ddots & \vdots \\
x_{n} x_{1}^{\nabla} & x_{n} x_{2}^{\nabla} & \cdots & x_{n} x_{n}^{\nabla}
\end{array}\right]\left[\begin{array}{c}
x_{1} \\
x_{2} \\
\vdots \\
x_{n}
\end{array}\right] \\
& =\left[\begin{array}{c}
x_{1}\left(x_{1} x_{1}^{\nabla}+x_{2} x_{2}^{\nabla}+\cdots+x_{n} x_{n}^{\nabla}\right) \\
x_{2}\left(x_{1} x_{1}^{\nabla}+x_{2} x_{2}^{\nabla}+\cdots+x_{n} x_{n}^{\nabla}\right) \\
\vdots \\
x_{n}\left(x_{1} x_{1}^{\nabla}+x_{2} x_{2}^{\nabla}+\cdots+x_{n} x_{n}^{\nabla}\right)
\end{array}\right] \\
& =\left[\begin{array}{c}
x_{1} \sum_{i=1}^{n} x_{i} x_{i}^{\nabla} \\
x_{2} \sum_{i=1}^{n} x_{i} x_{i}^{\nabla} \\
\vdots \\
x_{n} \sum_{i=1}^{n} x_{i} x_{i}^{\nabla}
\end{array}\right]=\sum_{i=1}^{n} x_{i} x_{i}^{\nabla}\left[\begin{array}{c}
x_{1} \\
x_{2} \\
\vdots \\
x_{n}
\end{array}\right] \\
& \rightarrow \boldsymbol{T}\left(\overrightarrow{\boldsymbol{x}}, \overrightarrow{\boldsymbol{x}}^{\nabla}\right) \overrightarrow{\boldsymbol{x}}=l \overrightarrow{\boldsymbol{x}}
\end{aligned}
$$

Such that $l=\sum_{i=1}^{n} x_{i} X_{i}^{\nabla}$ is equivalent to the digital level of the digital state $\hat{D}_{x} \overrightarrow{\boldsymbol{x}}$.

In tensor notation this equation is given as following:

$$
\boldsymbol{T}_{v}^{\mu} \boldsymbol{x}_{\mu}=l x_{\mu}
$$

\subsection{The Fluctuation Tensor Field}

The fluctuation tensor field is representing bilinear map [.,.]: $\mathbb{R}^{n} \times \mathbb{R}^{n} \rightarrow \mathbb{R}^{n \times n}$ -such that $\mathbb{R}^{n \times n}$ is the space of all $n \times n$ square matrix-defined for all $\boldsymbol{x}=\left(x_{1}, x_{2}, \cdots, x_{n}\right) \in \mathbb{R}^{n}$ and $\boldsymbol{y}=\left(y_{1}, y_{2}, \cdots, y_{n}\right) \in \mathbb{R}^{n}$ as following:

$$
[\boldsymbol{x}, \boldsymbol{y}]=\overrightarrow{\boldsymbol{y}} \otimes \overrightarrow{\boldsymbol{x}}-\overrightarrow{\boldsymbol{x}} \otimes \overrightarrow{\boldsymbol{y}}=\left[\begin{array}{cccc}
{[\boldsymbol{x}, \boldsymbol{y}]_{1,1}} & {[\boldsymbol{x}, \boldsymbol{y}]_{1,2}} & \cdots & {[\boldsymbol{x}, \boldsymbol{y}]_{1, n}} \\
{[\boldsymbol{x}, \boldsymbol{y}]_{2,1}} & {[\boldsymbol{x}, \boldsymbol{y}]_{2,2}} & \cdots & {[\boldsymbol{x}, \boldsymbol{y}]_{2, n}} \\
\vdots & \vdots & \ddots & \vdots \\
{[\boldsymbol{x}, \boldsymbol{y}]_{n, 1}} & {[\boldsymbol{x}, \boldsymbol{y}]_{n, 2}} & \cdots & {[\boldsymbol{x}, \boldsymbol{y}]_{n, n}}
\end{array}\right]
$$

Such that $\otimes$ is the outer product (tensor product) operation [2] defined as following:

$$
\overrightarrow{\boldsymbol{y}} \otimes \overrightarrow{\boldsymbol{x}}=\left[\begin{array}{c}
y_{1} \\
y_{2} \\
\vdots \\
y_{n}
\end{array}\right]\left[\begin{array}{llll}
x_{1} & x_{2} & \cdots & x_{n}
\end{array}\right]=\left[\begin{array}{cccc}
y_{1} x_{1} & y_{1} x_{2} & \cdots & y_{1} x_{n} \\
y_{2} x_{1} & y_{2} x_{2} & \cdots & y_{2} x_{n} \\
\vdots & \vdots & \ddots & \vdots \\
y_{n} x_{1} & y_{n} x_{2} & \cdots & y_{n} x_{n}
\end{array}\right],
$$

and

$$
\overrightarrow{\boldsymbol{x}} \otimes \overrightarrow{\boldsymbol{y}}=\left[\begin{array}{c}
x_{1} \\
x_{2} \\
\vdots \\
x_{n}
\end{array}\right]\left[\begin{array}{llll}
y_{1} & y_{2} & \cdots & y_{n}
\end{array}\right]=\left[\begin{array}{cccc}
x_{1} y_{1} & x_{1} y_{2} & \cdots & x_{1} y_{n} \\
x_{2} y_{1} & x_{2} y_{2} & \cdots & x_{2} y_{n} \\
\vdots & \vdots & \ddots & \vdots \\
x_{n} y_{1} & x_{n} y_{2} & \cdots & x_{n} y_{n}
\end{array}\right]
$$


Thus If $\boldsymbol{x}=\left(x_{1}, x_{2}, \cdots, x_{n}\right) \in \mathbb{R}^{n}$ and $\boldsymbol{y}=\left(y_{1}, y_{2}, \cdots, y_{n}\right) \in \mathbb{R}^{n}$ then the fluctuation tensor field of $x$ and $y$ donated by $[x, y]$ is the set of $n^{2}$ ordered real numbers $[x, y]_{\mu, v}$ called its components indexed by $\mu, v$ such that $\mu, v \in\{1,2, \cdots, n\}$ and defined as following:

$$
[\boldsymbol{x}, \boldsymbol{y}]_{\mu, v}=[\overrightarrow{\boldsymbol{x}}, \boldsymbol{y}]_{\mu, \nu}=[\boldsymbol{x}, \overrightarrow{\boldsymbol{y}}]_{\mu, v}=[\overrightarrow{\boldsymbol{x}}, \overrightarrow{\boldsymbol{y}}]_{\mu, \nu}=y_{\mu} x_{v}-x_{\mu} y_{\nu}
$$

\section{The Properties of commutator tensor field:}

a Anti-symmetric because:

$$
[x, y]=\vec{y} \otimes \vec{x}-\vec{x} \otimes \vec{y}=-(\vec{x} \otimes \vec{y}-\vec{y} \otimes \vec{x})-[y, x]
$$

b Alternating because:

$$
[\boldsymbol{x}, \boldsymbol{x}]=\overrightarrow{\boldsymbol{x}} \otimes \overrightarrow{\boldsymbol{x}}-\overrightarrow{\boldsymbol{x}} \otimes \overrightarrow{\boldsymbol{x}}=\left[\begin{array}{cccc}
0 & 0 & \cdots & 0 \\
0 & 0 & \cdots & 0 \\
\vdots & \vdots & \ddots & \vdots \\
0 & 0 & \cdots & 0
\end{array}\right] \in \mathbb{R}^{n \times n} .
$$

c Non-degenerate because:

For every $\boldsymbol{x}=\left(x_{1}, x_{2}, \cdots, x_{n}\right) \in \mathbb{R}^{n} /\{\boldsymbol{o}\}$ there exists $\boldsymbol{y}=\left(y_{1}, y_{2}, \cdots, y_{n}\right) \in \mathbb{R}^{n}$ and $\mu, v \in\{1,2, \cdots, n\}$ fulfills $x_{\mu} \neq 0, \quad y_{v} \neq 0$ and $\mu \neq v$ implied:

$$
[\boldsymbol{x}, \boldsymbol{y}] \neq\left[\begin{array}{cccc}
0 & 0 & \cdots & 0 \\
0 & 0 & \cdots & 0 \\
\vdots & \vdots & \ddots & \vdots \\
0 & 0 & \cdots & 0
\end{array}\right]
$$

Because $[\boldsymbol{x}, \boldsymbol{y}]_{\mu, v}=y_{\mu} x_{v}-y_{v} x_{\mu} \neq 0$.

d Bilinear because for all $\mu, v \in\{1,2, \cdots, n\}, \quad \boldsymbol{x}=\left(x_{1}, x_{2}, \cdots, x_{n}\right) \in \mathbb{R}^{n} /\{\boldsymbol{o}\}$, $\boldsymbol{y}=\left(y_{1}, y_{2}, \cdots, y_{n}\right) \in \mathbb{R}^{n} /\{\boldsymbol{o}\}, \quad \boldsymbol{z}=\left(z_{1}, z_{2}, \cdots, z_{n}\right) \in \mathbb{R}^{n}$ and all $a, b \in \mathbb{R}:$

$$
\begin{aligned}
{[a \boldsymbol{x}+b \boldsymbol{y}, \mathbf{z}]_{\mu, v} } & =z_{\mu}\left(a x_{v}+b y_{v}\right)-z_{v}\left(a x_{\mu}+b y_{\mu}\right) \\
& =a z_{\mu} x_{v}+b z_{\mu} y_{v}-a z_{v} x_{\mu}-b z_{v} y_{\mu} . \\
& =a[\boldsymbol{x}, \boldsymbol{y}]_{\mu, v}+b[\boldsymbol{y}, \mathbf{z}]_{\mu, v}
\end{aligned}
$$

Thus $[a \boldsymbol{x}+b \boldsymbol{y}, \mathbf{z}]=a[\boldsymbol{x}, \boldsymbol{y}]+b[\boldsymbol{y}, \mathbf{z}]$.

$$
[\boldsymbol{x}, a \boldsymbol{y}+b \mathbf{z}]_{\mu, v}=\left(a y_{\mu}+b z_{\mu}\right) x_{v}-\left(a y_{v}+b z_{v}\right) x_{\mu}
$$

Also

$$
\begin{aligned}
& =a y_{\mu} x_{v}+b z_{\mu} x_{v}-a y_{v} x_{\mu}-b z_{v} x_{\mu} . \\
& =a[\boldsymbol{x}, \boldsymbol{y}]_{\mu, v}+b[\boldsymbol{x}, \boldsymbol{z}]_{\mu, v}
\end{aligned}
$$

Thus $[\boldsymbol{x}, a \boldsymbol{y}+b \boldsymbol{z}]=a[\boldsymbol{x}, \boldsymbol{y}]+b[\boldsymbol{x}, \mathbf{z}]$.

\subsection{Theorem 3.3}

If $\boldsymbol{x}=\left(x_{1}, x_{2}, \cdots, x_{n}\right) \in \mathbb{R}^{n}, \boldsymbol{y}=\left(y_{1}, y_{2}, \cdots, y_{n}\right) \in \mathbb{R}^{n}$ then the fluctuation tensor $[\boldsymbol{x}, \boldsymbol{y}]$ is representing a measure of degree to which the point $\boldsymbol{x}$ and $\boldsymbol{y}$ deviate from belonging to the collinear set $\mathbb{R}^{n}(\boldsymbol{o}, \boldsymbol{y})$ and $\mathbb{R}^{n}(\boldsymbol{o}, \boldsymbol{x})$ respectively because:

1) $[\boldsymbol{x}, \boldsymbol{y}]=\mathbf{0}$ for all $\boldsymbol{p} \in \mathbb{R}^{n}(\boldsymbol{o}, \boldsymbol{x})$ vanished when there is no deviation.

2) $[x, y]=[x, y+\overrightarrow{d x}]=[x+\overrightarrow{d y}, y]$ for all $\overrightarrow{\boldsymbol{d x}} \in \overrightarrow{\mathbb{R}^{n}}(o, x)$ and 
$\overrightarrow{\boldsymbol{d y}} \in \overrightarrow{\mathbb{R}^{n}}(\boldsymbol{o}, \boldsymbol{y})$ i.e. the fluctuation tensor field $[\boldsymbol{x}, \boldsymbol{y}]$ is a invariant under parallel translation of $\boldsymbol{x}$ with respect to the line that all elements of $\mathbb{R}^{n}(\boldsymbol{o}, \boldsymbol{y})$ lie or parallel translation of $y$ with respect to the line that all elements of $\mathbb{R}^{n}(\boldsymbol{o}, \boldsymbol{x})$ lie. Thus all element lie at single line parallel to the line that all elements of some collinear set $S$ lie have a same measure of deviation from belonging to $S$ defined as fluctuation tensor field.

Prove:

1) for all $\boldsymbol{p}=\left(p_{1}, p_{2}, \cdots, p_{n}\right) \in \mathbb{R}^{n}(\boldsymbol{o}, \boldsymbol{x})$ and all $i \in\{1,2, \cdots, n\}$ fulfill $x_{i} \neq 0$ :

$$
\boldsymbol{p}=\frac{x_{i}-p_{i}}{x_{i}-0} \boldsymbol{o}+\frac{p_{i}-0}{x_{i}-0} \boldsymbol{x}=\frac{p_{i}}{x_{i}} \boldsymbol{x}
$$

and hence:

$$
\begin{aligned}
& {[\boldsymbol{x}, \boldsymbol{p}]_{\mu, v}=\left[\boldsymbol{x}, \frac{p_{i}}{x_{i}} \boldsymbol{p}\right]_{\mu, v}=\frac{p_{i}}{x_{i}} x_{\mu} x_{v}-x_{\mu} \frac{p_{i}}{x_{i}} x_{v}=\frac{p_{i}}{x_{i}}\left(x_{\mu} x_{v}-x_{\mu} x_{v}\right)=0 .} \\
& \rightarrow[x, p]=0 . \\
& \text { 2) For all } \overrightarrow{\boldsymbol{d}}=\left[\begin{array}{c}
d x_{1} \\
d x_{2} \\
\vdots \\
d x_{n}
\end{array}\right] \in \mathbb{R}^{n}, \overrightarrow{\boldsymbol{d y}}=\left[\begin{array}{c}
d y_{1} \\
d y_{2} \\
\vdots \\
d y_{n}
\end{array}\right] \in \mathbb{R}^{n} \text { and all } \mu, v \in\{1,2, \cdots, n\} \text { : } \\
& {[\boldsymbol{x}, \boldsymbol{y}+\overrightarrow{\boldsymbol{d} \boldsymbol{x}}]_{\mu, v}=\left(y_{\mu}+d x_{\mu}\right) x_{v}-\left(y_{v}+d x_{v}\right) x_{\mu}} \\
& =y_{\mu} x_{v}+x_{v} d x_{\mu}-y_{v} x_{\mu}-x_{\mu} d x_{v} \\
& =[\boldsymbol{x}, \boldsymbol{y}]_{\mu, v}+[\boldsymbol{x}, \overrightarrow{\boldsymbol{d x}}]_{\mu, v} \text {. } \\
& {[\boldsymbol{x}+\overrightarrow{\boldsymbol{d}}, \boldsymbol{y}]_{\mu, v}=y_{\mu}\left(x_{v}+d y_{v}\right)-y_{v}\left(x_{\mu}+d y_{\mu}\right)} \\
& =y_{\mu} x_{v}+y_{\mu} d y_{v}-y_{v} x_{\mu}-y_{v} d y_{\mu} \\
& =[\boldsymbol{x}, \boldsymbol{y}]_{\mu, v}+[\boldsymbol{y}, \overrightarrow{\boldsymbol{d y}}]_{\mu, v}
\end{aligned}
$$

Thus if $\overrightarrow{\boldsymbol{d x}} \in \overrightarrow{\mathbb{R}^{n}}(\boldsymbol{o}, \boldsymbol{x})$ and $\overrightarrow{\boldsymbol{d y}} \in \overrightarrow{\mathbb{R}^{n}}(\boldsymbol{o}, \boldsymbol{y})$ then there exist four points $\boldsymbol{p}=\left(p_{1}, p_{2}, \cdots, p_{n}\right) \in \mathbb{R}^{n}(\boldsymbol{o}, \boldsymbol{x}), \quad \boldsymbol{q}=\left(q_{1}, q_{2}, \cdots, q_{n}\right) \in \mathbb{R}^{n}(\boldsymbol{o}, \boldsymbol{x})$, $\boldsymbol{r}=\left(r_{1}, r_{2}, \cdots, r_{n}\right) \in \mathbb{R}^{n}(\boldsymbol{o}, \boldsymbol{y}), \boldsymbol{s}=\left(s_{1}, s_{2}, \cdots, s_{n}\right) \in \mathbb{R}^{n}(\boldsymbol{o}, \boldsymbol{x})$ fulfill $\overrightarrow{\boldsymbol{d} \boldsymbol{x}}=\overrightarrow{\boldsymbol{d}}(\boldsymbol{p}, \boldsymbol{q})=\boldsymbol{q}-\boldsymbol{p}, \overrightarrow{\boldsymbol{d} \boldsymbol{y}}=\overrightarrow{\boldsymbol{d}}(\boldsymbol{r}, \boldsymbol{s})=\boldsymbol{s}-\boldsymbol{r}$ and for all $i \in\{1,2, \cdots, n\}$ fulfill $x_{i} \neq 0$ and $q_{i} \neq p_{i}$ and all $j \in\{1,2, \cdots, n\}$ fulfill $y_{j} \neq 0$ and $r_{j} \neq s_{j}$ :

$$
\begin{aligned}
& \boldsymbol{p}=\frac{x_{i}-p_{i}}{x_{i}-0} \boldsymbol{o}+\frac{p_{i}-0}{x_{i}-0} \boldsymbol{x}=\frac{p_{i}}{x_{i}} \boldsymbol{x}, \\
& \boldsymbol{q}=\frac{x_{i}-q_{i}}{x_{i}-0} \boldsymbol{o}+\frac{q_{i}-0}{x_{i}-0} \boldsymbol{x}=\frac{q_{i}}{x_{i}} \boldsymbol{x}, \\
& \boldsymbol{r}=\frac{y_{j}-r_{j}}{y_{j}-0} \boldsymbol{o}+\frac{r_{j}-0}{y_{j}-0} \boldsymbol{y}=\frac{r_{j}}{y_{i}} \boldsymbol{y}, \\
& \boldsymbol{s}=\frac{y_{j}-s_{j}}{y_{j}-0} \boldsymbol{o}+\frac{s_{j}-0}{y_{j}-0} \boldsymbol{y}=\frac{s_{j}}{y_{i}} \boldsymbol{y}, \\
& \rightarrow \overrightarrow{\boldsymbol{d} \boldsymbol{x}}=\overrightarrow{\boldsymbol{d}}(\boldsymbol{p}, \boldsymbol{q})=\boldsymbol{q}-\boldsymbol{p}=\frac{q_{i}}{x_{i}} \boldsymbol{x}-\frac{p_{i}}{x_{i}} \boldsymbol{x}=\left(\frac{q_{i}-p_{i}}{x_{i}}\right) \boldsymbol{x}
\end{aligned}
$$




$$
\overrightarrow{\boldsymbol{d} \boldsymbol{y}}=\overrightarrow{\boldsymbol{d}}(\boldsymbol{r}, \boldsymbol{s})=\boldsymbol{s}-\boldsymbol{r}=\frac{s_{j}}{y_{j}} \boldsymbol{y}-\frac{r_{j}}{y_{j}} \boldsymbol{y}=\left(\frac{s_{j}-r_{j}}{y_{j}}\right) \boldsymbol{y} \rightarrow
$$

For all $\mu, v \in\{1,2, \cdots, n\}$ :

$$
\begin{aligned}
& {[\boldsymbol{x}, \boldsymbol{y}+\overrightarrow{\boldsymbol{d} \boldsymbol{x}}]_{\mu, \nu}=[\boldsymbol{x}, \boldsymbol{y}]_{\mu, \nu}+[\boldsymbol{x}, \overrightarrow{\boldsymbol{d} \boldsymbol{x}}]_{\mu, \nu}=[\boldsymbol{x}, \boldsymbol{y}]_{\mu, \nu}+\left[\boldsymbol{x},\left(\frac{q_{i}-p_{i}}{x_{i}}\right) \boldsymbol{x}\right]_{\mu, \nu}} \\
& =[\boldsymbol{x}, \boldsymbol{y}]_{\mu, v}+\left(\frac{q_{i}-p_{i}}{x_{i}}\right)[\boldsymbol{x}, \boldsymbol{x}]_{\mu, v}=[\boldsymbol{x}, \boldsymbol{y}]_{\mu, v} \\
& {[\boldsymbol{x}+\overrightarrow{\boldsymbol{d} y}, \boldsymbol{y}]_{\mu, v}=[\boldsymbol{x}, \boldsymbol{y}]_{\mu, v}+[\boldsymbol{y}, \overrightarrow{\boldsymbol{d} y}]_{\mu, v}=[\boldsymbol{x}, \boldsymbol{y}]_{\mu, \nu}+\left[\boldsymbol{y},\left(\frac{s_{j}-r_{j}}{y_{j}}\right) \boldsymbol{y}\right]_{\mu, \nu}} \\
& =[\boldsymbol{x}, \boldsymbol{y}]_{\mu, v}+\left(\frac{s_{j}-r_{j}}{y_{j}}\right)[\boldsymbol{y}, \boldsymbol{y}]_{\mu, v}=[\boldsymbol{x}, \boldsymbol{y}]_{\mu, v} \\
& \rightarrow[x, y+\overrightarrow{d x}]=[x+\overrightarrow{d y}, y]=[x, y] .
\end{aligned}
$$

\subsection{The Spin's Fluctuation Tensor Field at Each Matter Particle's Surface Point}

The spin fluctuation tensor field is defined at each surface point of any matter particle resisting with respect to an arbitrary observer at the position $\overrightarrow{\boldsymbol{r}}=\left[\begin{array}{l}x \\ y \\ z\end{array}\right]$ as following:

$$
[\overrightarrow{\boldsymbol{r}}, \overrightarrow{\boldsymbol{p}}]=\left[\begin{array}{ccc}
0 & y p_{x}-x p_{y} & z p_{x}-x p_{z} \\
x p_{y}-y p_{x} & 0 & z p_{y}-y p_{z} \\
x p_{z}-z p_{x} & y p_{z}-z p_{y} & 0
\end{array}\right]
$$

where: $\overrightarrow{\boldsymbol{p}}=\left[\begin{array}{c}p_{x} \\ p_{y} \\ p_{z}\end{array}\right]$ is linear and momentum vector of the matter particle.

\section{Important note:}

Each components of the spin's fluctuation tensor field $[\overrightarrow{\boldsymbol{r}}, \overrightarrow{\boldsymbol{p}}]$ is corresponding to either positive or negative components of angular momentum vector:

$\overrightarrow{\boldsymbol{L}}=\overrightarrow{\boldsymbol{r}} \times \overrightarrow{\boldsymbol{p}}=$ Determinat $\left(\left[\begin{array}{ccc}\boldsymbol{i} & \boldsymbol{j} & \boldsymbol{k} \\ x & y & z \\ p_{x} & p_{y} & p_{z}\end{array}\right]\right)=\left[\begin{array}{c}y p_{z}-z p_{y} \\ z p_{x}-x p_{z} \\ x p_{y}-y p_{x}\end{array}\right]$ and vice versa, so the components of the spin's fluctuation tensor field $\mathcal{F}(\overrightarrow{\boldsymbol{r}}, \overrightarrow{\boldsymbol{p}})$ are vanishesbecomes zero-when all component of $\overrightarrow{\boldsymbol{L}}$ are vanishes and vice versa, also the components of the spin's fluctuation tensor field $[\overrightarrow{\boldsymbol{r}}, \overrightarrow{\boldsymbol{p}}]$ are conserved when the components of $\overrightarrow{\boldsymbol{L}}$ are conserved and vice versa, those two strong correlated feature between the components of the spin's fluctuation tensor field $[\overrightarrow{\boldsymbol{r}}, \overrightarrow{\boldsymbol{p}}]$ and the components of $\overrightarrow{\boldsymbol{L}}$ allow the author to introduce the following theorem. 


\subsection{Theorem 3.5: Spin's Fluctuation Theorem}

Any matter particle $P$ possesses a non-zero mass $m$ can possess:

1) A non-zero spin angular momentum at arbitrary time interval $[t, t+d t]$ if and only if all surface points of $P$ are not moving in the same direction of its position vector during this time interval.

2) A conserved spin angular momentum at any two arbitrary time intervals $[t, t+d t]$ and $\left[t+d t, t+d t+d t^{\prime}\right]$ if and only if any surface points of $P$ donated by $s$ located with respect to an arbitrary observer or measurement instruement at the position $\overrightarrow{\boldsymbol{r}}=\left[\begin{array}{l}x \\ y \\ z\end{array}\right]$ at the time $t$ fulfills:

$$
\left[\overrightarrow{\boldsymbol{r}}+\overrightarrow{\boldsymbol{d} \boldsymbol{r}}, \overrightarrow{\boldsymbol{r}}+\overrightarrow{\boldsymbol{d} \boldsymbol{r}}+\overrightarrow{\boldsymbol{d} \boldsymbol{r}^{\prime}}\right] d t^{\prime}=[\overrightarrow{\boldsymbol{r}}, \boldsymbol{r}+\overrightarrow{\boldsymbol{d} \boldsymbol{r}}] d t
$$

where:

i) $\overrightarrow{\boldsymbol{d r}}$ is infinitesimal displacement of $s$ at the time interval $[t, t+d t]$.

ii) $\overrightarrow{\boldsymbol{d r}^{\prime}}$ is infinitesimal displacement of $s$ at the time interval $\left[t+d t, t+d t+d t^{\prime}\right]$.

\section{Prove:}

1) Suppose we have some arbitrary surface point of $P$ resisting with respect to an arbitrary observer at the position $\overrightarrow{\boldsymbol{r}}=\left[\begin{array}{l}x \\ y \\ z\end{array}\right]$ at the time $t$.

Now the spin angular momentum of $P$ at $\overrightarrow{\boldsymbol{r}}$ is defined as a cross product of $\overrightarrow{\boldsymbol{r}}$ and the linear momentum vector $\overrightarrow{\boldsymbol{p}}=\left[\begin{array}{c}p_{x} \\ p_{y} \\ p_{z}\end{array}\right]=m\left[\begin{array}{c}\frac{d x}{d t} \\ \frac{d y}{d t} \\ \frac{d z}{d t}\end{array}\right]$ as following:

$$
\overrightarrow{\boldsymbol{L}}=\overrightarrow{\boldsymbol{r}} \times \overrightarrow{\boldsymbol{p}}=\left|\begin{array}{ccc}
\boldsymbol{i} & \boldsymbol{j} & \boldsymbol{k} \\
x & y & z \\
p_{x} & p_{y} & p_{z}
\end{array}\right|=\left[\begin{array}{c}
y p_{z}-z p_{y} \\
z p_{x}-x p_{z} \\
x p_{y}-y p_{x}
\end{array}\right]
$$

In other hand the spin's fluctuation tensor field $[\overrightarrow{\boldsymbol{r}}, \overrightarrow{\boldsymbol{p}}]$ is defined as following:

$$
[\overrightarrow{\boldsymbol{r}}, \overrightarrow{\boldsymbol{p}}]=\left[\begin{array}{ccc}
0 & y p_{x}-x p_{y} & z p_{x}-x p_{z} \\
x p_{y}-y p_{x} & 0 & z p_{y}-y p_{z} \\
x p_{z}-z p_{x} & y p_{z}-z p_{y} & 0
\end{array}\right]
$$

Thus every component of $\overrightarrow{\boldsymbol{L}}$ is equivalent to either positive or negative value of one component of $[\overrightarrow{\boldsymbol{r}}, \overrightarrow{\boldsymbol{p}}]$, so all components of $\overrightarrow{\boldsymbol{L}}$ vanishes-becomes zero-when all components of $[\overrightarrow{\boldsymbol{r}}, \overrightarrow{\boldsymbol{p}}]$ vanishes.

Now the infinitesimal displacement vector $\overrightarrow{\boldsymbol{d r}}=\left[\begin{array}{l}d x \\ d y \\ d z\end{array}\right]$, thus the fluctuation 
tensor field of $\overrightarrow{\boldsymbol{r}}$ and $\overrightarrow{\boldsymbol{r}}+\overrightarrow{\boldsymbol{d} \boldsymbol{r}}$ is defined using bilinearity property of fluctuation tensor field as following:

$$
\begin{aligned}
{[\overrightarrow{\boldsymbol{r}}, \overrightarrow{\boldsymbol{r}}+\overrightarrow{\boldsymbol{d r}}] } & =[\overrightarrow{\boldsymbol{r}}, \overrightarrow{\boldsymbol{r}}]+[\overrightarrow{\boldsymbol{r}}, \overrightarrow{\boldsymbol{d} \boldsymbol{r}}]=[\overrightarrow{\boldsymbol{r}}, \overrightarrow{\boldsymbol{d} r}]=\left[\overrightarrow{\boldsymbol{r}}, \frac{d t}{m} \overrightarrow{\boldsymbol{p}}\right]=\frac{d t}{m}[\overrightarrow{\boldsymbol{r}}, \overrightarrow{\boldsymbol{p}}] \\
& \rightarrow[\overrightarrow{\boldsymbol{r}}, \overrightarrow{\boldsymbol{p}}]=\frac{d t}{m}[\overrightarrow{\boldsymbol{r}}, \overrightarrow{\boldsymbol{r}}+\overrightarrow{\boldsymbol{d} \boldsymbol{r}}]=\frac{d t}{m}[\overrightarrow{\boldsymbol{r}}, \overrightarrow{\boldsymbol{d} \boldsymbol{r}}]
\end{aligned}
$$

Because $d t$ is representing infinitesimal non-zero change of time, the spin's fluctuation tensor field $[\overrightarrow{\boldsymbol{r}}, \overrightarrow{\boldsymbol{p}}]$ vanishes-and then the spin angular momentum vector $\overrightarrow{\boldsymbol{L}}$-when all components of the fluctuation tensor field $[\overrightarrow{\boldsymbol{r}}, \overrightarrow{\boldsymbol{r}}+\overrightarrow{\boldsymbol{d r}}]=[\overrightarrow{\boldsymbol{r}}, \overrightarrow{\boldsymbol{d r}}]$ vanishes so according to the theorem $3.2 \overrightarrow{\boldsymbol{L}}$ vanishes when $\overrightarrow{\boldsymbol{r}}+\overrightarrow{\boldsymbol{d r}} \in \mathbb{R}^{3}(\boldsymbol{o}, \boldsymbol{r})$ or equivalently when $\overrightarrow{\boldsymbol{d r}} \in \overrightarrow{\mathbb{R}^{3}}(\boldsymbol{o}, \boldsymbol{r})$. Thus the matter particle $P$ possesses a non-zero spin angular momentum if and only if all surface points of $P$ are not moving in the same direction of their position vectors.

2) Let $s$ represents a surface point of $P$ resisting with respect to an arbitrary observer at the position $\overrightarrow{\boldsymbol{r}}=\left[\begin{array}{c}x \\ y \\ z\end{array}\right]$ at the time $t$, let $\overrightarrow{\boldsymbol{d} \boldsymbol{r}}=\left[\begin{array}{l}d x \\ d y \\ d z\end{array}\right]=\frac{d t}{m} \overrightarrow{\boldsymbol{p}}=\frac{d t}{m}\left[\begin{array}{l}p_{x} \\ p_{y} \\ p_{z}\end{array}\right]$ is representing the infinitesimal displacement of the matter particle's surface points during the time interval $[t, t+d t], \overrightarrow{r^{\prime}}=\left[\begin{array}{c}x+d x \\ y+d y \\ z+d z\end{array}\right]=\left[\begin{array}{c}x^{\prime} \\ y^{\prime} \\ z^{\prime}\end{array}\right]$ is the position of the matter particle's surface point $s$ at the time $t+d t$, and let $\overrightarrow{\boldsymbol{d r}^{\prime}}=\left[\begin{array}{l}d x^{\prime} \\ d y^{\prime} \\ d z^{\prime}\end{array}\right]=\frac{d t^{\prime}}{m} \overrightarrow{\boldsymbol{p}^{\prime}}=\frac{d t^{\prime}}{m}\left[\begin{array}{l}p_{x}^{\prime} \\ p_{y}^{\prime} \\ p_{z}^{\prime}\end{array}\right]$ is representing the infinitesimal displacement of the matter particle's surface point $s$ during the time interval $\left[t+d t, t+d t+d t^{\prime}\right]$, now the spin angular momentum vectors of the matter particle at $s$ during the time interval $[t, t+d t]$ and $\left[t+d t, t+d t+d t^{\prime}\right]$ and donated by $\overrightarrow{\boldsymbol{L}}$ and $\overrightarrow{\boldsymbol{L}^{\prime}}$ respectively are given as following:

$$
\begin{gathered}
\overrightarrow{\boldsymbol{L}}=\overrightarrow{\boldsymbol{r}} \times \overrightarrow{\boldsymbol{p}}=\text { Determinat }\left(\left[\begin{array}{ccc}
\boldsymbol{i} & \boldsymbol{j} & \boldsymbol{k} \\
x & y & z \\
p_{x} & p_{y} & p_{z}
\end{array}\right]\right)=\left[\begin{array}{c}
y p_{z}-z p_{y} \\
z p_{x}-x p_{z} \\
x p_{y}-y p_{x}
\end{array}\right] \\
\overrightarrow{\boldsymbol{L}^{\prime}}=\overrightarrow{\boldsymbol{r}^{\prime}} \times \overrightarrow{\boldsymbol{p}^{\prime}}=\text { Determinat }\left(\left[\begin{array}{ccc}
\boldsymbol{i} & \boldsymbol{j} & \boldsymbol{k} \\
x^{\prime} & y^{\prime} & z^{\prime} \\
p_{x}^{\prime} & p_{y}^{\prime} & p_{z}^{\prime}
\end{array}\right]\right)=\left[\begin{array}{c}
y^{\prime} p_{z}^{\prime}-z^{\prime} p_{y}^{\prime} \\
z^{\prime} p_{x}^{\prime}-x^{\prime} p_{z}^{\prime} \\
x^{\prime} p_{y}^{\prime}-y^{\prime} p_{x}^{\prime}
\end{array}\right]
\end{gathered}
$$

Also the spin's fluctuations tensor fields of the matter particle at $s$ during the time interval $[t, t+d t]$ and $\left[t+d t, t+d t+d t^{\prime}\right]$ and donated by $[\overrightarrow{\boldsymbol{r}}, \overrightarrow{\boldsymbol{p}}]$ and $\left[\overrightarrow{\boldsymbol{r}^{\prime}}, \overrightarrow{\boldsymbol{p}^{\prime}}\right]$ respectively are given as following:

$$
[\overrightarrow{\boldsymbol{r}}, \overrightarrow{\boldsymbol{p}}]=\left[\begin{array}{ccc}
0 & y p_{x}-x p_{y} & z p_{x}-x p_{z} \\
x p_{y}-y p_{x} & 0 & z p_{y}-y p_{z} \\
x p_{z}-z p_{x} & y p_{z}-z p_{y} & 0
\end{array}\right]
$$




$$
\left[\overrightarrow{\boldsymbol{r}^{\prime}}, \overrightarrow{\boldsymbol{p}^{\prime}}\right]=\left[\begin{array}{ccc}
0 & y^{\prime} p_{x}^{\prime}-x^{\prime} p_{y}^{\prime} & z^{\prime} p_{x}^{\prime}-x^{\prime} p_{z}^{\prime} \\
x^{\prime} p_{y}^{\prime}-y^{\prime} p_{x}^{\prime} & 0 & z^{\prime} p_{y}^{\prime}-y^{\prime} p_{z}^{\prime} \\
x^{\prime} p_{z}^{\prime}-z^{\prime} p_{x}^{\prime} & y^{\prime} p_{z}^{\prime}-z^{\prime} p_{y}^{\prime} & 0
\end{array}\right]
$$

Thus every component of $\overrightarrow{\boldsymbol{L}}$ is equivalent to either positive or negative value of one component of $[\overrightarrow{\boldsymbol{r}}, \overrightarrow{\boldsymbol{p}}]$ and every component of $\overrightarrow{\boldsymbol{L}}^{\prime}$ is equivalent to either positive or negative value of one component of $\left[\overrightarrow{\boldsymbol{r}^{\prime}}, \overrightarrow{\boldsymbol{p}^{\prime}}\right]$, so $\overrightarrow{\boldsymbol{L}}=\overrightarrow{\boldsymbol{L}^{\prime}}$ if and only if $[\overrightarrow{\boldsymbol{r}}, \overrightarrow{\boldsymbol{p}}]=\left[\overrightarrow{\boldsymbol{r}^{\prime}}, \overrightarrow{\boldsymbol{p}^{\prime}}\right]$ and then if and only if:

$$
\left[\overrightarrow{\boldsymbol{r}}, \frac{d t}{m} \overrightarrow{\boldsymbol{d} \boldsymbol{r}}\right]=\left[\overrightarrow{\boldsymbol{r}}+\overrightarrow{\boldsymbol{d} \boldsymbol{r}}, \frac{d t^{\prime}}{m} \overrightarrow{\boldsymbol{d} \boldsymbol{r}^{\prime}}\right]
$$

or equavently if and only if:

$$
[\overrightarrow{\boldsymbol{r}}, \overrightarrow{\boldsymbol{d} \boldsymbol{r}}] d t=\left[\overrightarrow{\boldsymbol{r}}+\overrightarrow{\boldsymbol{d r}}, \overrightarrow{\boldsymbol{d} \boldsymbol{r}^{\prime}}\right] d t^{\prime}
$$

or equivently if and only if:

Because

$$
[\vec{r}, \boldsymbol{r}+\overrightarrow{d r}]=[\vec{r}, \vec{r}]+[\vec{r}, \overrightarrow{d r}]=[\vec{r}, \overrightarrow{d r}]
$$

and

$$
\begin{gathered}
{\left[\overrightarrow{\boldsymbol{r}}+\overrightarrow{\boldsymbol{d r}}, \overrightarrow{\boldsymbol{r}}+\overrightarrow{\boldsymbol{d r}}+\overrightarrow{\boldsymbol{d r}} \boldsymbol{r}^{\prime}\right]=[\overrightarrow{\boldsymbol{r}}+\overrightarrow{\boldsymbol{d r}}, \overrightarrow{\boldsymbol{r}}+\overrightarrow{\boldsymbol{d r}}]+\left[\overrightarrow{\boldsymbol{r}}+\overrightarrow{\boldsymbol{d r}}, \overrightarrow{\boldsymbol{d r}} \boldsymbol{r}^{\prime}\right]=\left[\overrightarrow{\boldsymbol{r}}+\overrightarrow{\boldsymbol{d r}}, \overrightarrow{\boldsymbol{d r}} \vec{r}^{\prime}\right],} \\
{[\overrightarrow{\boldsymbol{r}}, \overrightarrow{\boldsymbol{p}}]=\left[\overrightarrow{\boldsymbol{r}^{\prime}}, \overrightarrow{p^{\prime}}\right]}
\end{gathered}
$$

if and only if:

$$
\left[\overrightarrow{\boldsymbol{r}}+\overrightarrow{\boldsymbol{d} \boldsymbol{r}}, \overrightarrow{\boldsymbol{r}}+\overrightarrow{\boldsymbol{d} \boldsymbol{r}}+\overrightarrow{\boldsymbol{d} \boldsymbol{r}^{\prime}}\right] d t^{\prime}=[\overrightarrow{\boldsymbol{r}}, \boldsymbol{r}+\overrightarrow{\boldsymbol{d} \boldsymbol{r}}] d t
$$

\section{Important Notes:}

1) The first part of the above theorem implied that if the matter particle possesses non-zero mass and non-zero spin angular momentum then it's all surface points should move in different direction of all position vectors defined with respect to all observers observing them, thus the first part of the above theorem approve that the non-zero spin angular momentum of any matter particle possesses non-zero mass is intrinsic property independent from the observer's localization with respect to its surface point.

2) The second part of the above theorem illustrate the relation between spatial and temporal coordinates of the surface points of any matter particle possesses non-zero mass and conserved spin angular momentum.

\subsection{Theorem 3.6}

If $\boldsymbol{x}=\left(x_{1}, x_{2}, \cdots, x_{n}\right) \in \mathbb{R}^{n}, \quad \boldsymbol{y}=\left(y_{1}, y_{2}, \cdots, y_{n}\right) \in \mathbb{R}^{n}, \quad \boldsymbol{z}=\left(z_{1}, z_{2}, \cdots, z_{n}\right) \in \mathbb{R}^{n}$ and $\boldsymbol{w}=\left(w_{1}, w_{2}, \cdots, w_{n}\right) \in \mathbb{R}^{n}$ such that $\boldsymbol{x} \neq \boldsymbol{y}, \boldsymbol{w} \neq \mathbf{z}, \mathbb{R}^{n}(\boldsymbol{x}, \boldsymbol{y})$ is not parallel to $\mathbb{R}^{n}(\mathbf{z}, \boldsymbol{w})$ and $\mathbb{R}^{n}(\boldsymbol{x}, \boldsymbol{y}) \cap \mathbb{R}^{n}(\boldsymbol{z}, \boldsymbol{w}) \notin\{\boldsymbol{x}, \boldsymbol{y}, \boldsymbol{z}, \boldsymbol{w}\}$ then $\mathbb{R}^{n}(\boldsymbol{x}, \boldsymbol{y})$ and $\mathbb{R}^{n}(\mathbf{z}, \boldsymbol{w})$ are intersected at the point $\boldsymbol{t}=\left(t_{1}, t_{2}, \cdots, t_{n}\right) \in \mathbb{R}^{n}$ fulfills:

1)

$$
t_{\varphi}=\frac{[\vec{d}(x, y), \vec{d}(z, w)]_{\mu, v}[x, y]_{\mu, \varphi}+[x, y]_{\mu, v} d_{\mu}(z, w) d_{\varphi}(x, y)-[z, w]_{\mu, v} d_{\mu}(x, y) d_{\varphi}(x, y)}{[\vec{d}(x, y), \vec{d}(z, w)]_{\mu, v} d_{\mu}(x, y)}
$$


For all $\varphi \in\{1,2, \cdots, n\}$ and all $\mu, v \in\{1,2, \cdots, n\}$ fulfill $[\vec{d}(x, y), \vec{d}(z, w)]_{\mu, v} d_{\mu}(x, y) \neq 0$.

2) if $\boldsymbol{x}=\boldsymbol{t}+a \overrightarrow{\boldsymbol{d} t}, \boldsymbol{y}=\boldsymbol{t}+b \overrightarrow{\boldsymbol{d t}}, \mathbf{z}=\boldsymbol{t}+a^{\prime} \overrightarrow{\boldsymbol{d t}^{\prime}}$ and $\boldsymbol{w}=\boldsymbol{t}+b^{\prime} \overrightarrow{\boldsymbol{d t}^{\prime}}$ for some $a, b, a^{\prime}, b^{\prime} \in \mathbb{R} /\{0\}$ fulfill $a \neq b$ and $a^{\prime} \neq b^{\prime}$, and some $\overrightarrow{\boldsymbol{d} t}=\left[\begin{array}{c}d t_{1} \\ d t_{2} \\ \vdots \\ d t_{n}\end{array}\right] \in \mathbb{R}^{n}$ and $\overrightarrow{\mathbf{d t}^{\prime}}=\left[\begin{array}{c}d t_{1}^{\prime} \\ d t_{2}^{\prime} \\ \vdots \\ d t_{n}^{\prime}\end{array}\right]$ fulfill $d t_{\mu} \neq 0, d t_{v}^{\prime} \neq 0, \overrightarrow{\mathbf{d t}^{\prime}} \neq \lambda \overrightarrow{\mathbf{d t}}$ for all $\lambda \in \mathbb{R}-i . e . \overrightarrow{\mathbf{d t}^{\prime}}$ is not parallel to $\overrightarrow{\boldsymbol{d} t}$-and either $d t_{v} \neq 0$ or $d t_{\mu}^{\prime} \neq 0$, then:

$$
\left[\overrightarrow{\boldsymbol{d} t}, \overrightarrow{\mathbf{d} \boldsymbol{t}^{\prime}}\right]_{\mu, \nu}\left(t_{\varphi} d t_{\mu}-[\boldsymbol{t}, \overrightarrow{\boldsymbol{d} t}]_{\mu, \varphi}\right)=[\boldsymbol{t}, \overrightarrow{\boldsymbol{d} t}]_{\mu, \nu} d t_{\mu}^{\prime} d t_{\varphi}-\left[\boldsymbol{t}, \overrightarrow{\boldsymbol{d} \boldsymbol{t}^{\prime}}\right]_{\mu, \nu} d t_{\mu} d t_{\varphi} .
$$

For all $\varphi \in\{1,2, \cdots, n\}$ and all $\mu, v \in\{1,2, \cdots, n\}$ fulfill $\left[\overrightarrow{\boldsymbol{d t}}, \overrightarrow{\boldsymbol{d t}^{\prime}}\right]_{\mu, \nu} d t_{\mu} \neq 0$.

\section{Prove:}

Suppose we have $\boldsymbol{x}=\left(x_{1}, x_{2}, \cdots, x_{n}\right) \in \mathbb{R}^{n}, \quad \boldsymbol{y}=\left(y_{1}, y_{2}, \cdots, y_{n}\right) \in \mathbb{R}^{n}$, $\boldsymbol{z}=\left(z_{1}, z_{2}, \cdots, z_{n}\right) \in \mathbb{R}^{n}$ and $\boldsymbol{w}=\left(w_{1}, w_{2}, \cdots, w_{n}\right) \in \mathbb{R}^{n}$ such that $\boldsymbol{x} \neq \boldsymbol{y}, \boldsymbol{w} \neq \mathbf{z}$, $\mathbb{R}^{n}(\boldsymbol{x}, \boldsymbol{y}) \cap \mathbb{R}^{n}(\mathbf{z}, \boldsymbol{w}) \notin\{\boldsymbol{x}, \boldsymbol{y}, \mathbf{z}, \boldsymbol{w}\}$ and $\mathbb{R}^{n}(\boldsymbol{x}, \boldsymbol{y})$ is not parallel to $\mathbb{R}^{n}(\boldsymbol{z}, \boldsymbol{w})$, suppose that $\boldsymbol{t}=\left(t_{1}, t_{2}, \cdots, t_{n}\right) \in \mathbb{R}^{n}$ is intersection point of $\mathbb{R}^{n}(\boldsymbol{x}, \boldsymbol{y})$ and $\mathbb{R}^{n}(\mathbf{z}, \boldsymbol{w})$ : Now for all $\mu, v \in\{1,2, \cdots, n\}$ fulfills $x_{\mu} \neq y_{\mu}, \quad z_{v} \neq w_{v}$ and either $x_{v} \neq y_{v}$ or $z_{\mu} \neq w_{\mu}-$ i.e. $[\boldsymbol{d}(\boldsymbol{x}, \boldsymbol{y}), \boldsymbol{d}(\mathbf{z}, \boldsymbol{w})]_{\mu, v} \neq 0$ :

$$
\frac{y_{\mu}-t_{\mu}}{y_{\mu}-x_{\mu}} \boldsymbol{x}+\frac{t_{\mu}-x_{\mu}}{y_{\mu}-x_{\mu}} \boldsymbol{y}=\left(t_{1}, t_{2}, \cdots, t_{n}\right)
$$

and

$$
\begin{gathered}
\frac{w_{v}-t_{v}}{w_{v}-z_{v}} \mathbf{Z}+\frac{t_{v}-z_{v}}{w_{v}-z_{v}} \boldsymbol{w}=\left(t_{1}, t_{2}, \cdots, t_{n}\right) \\
\rightarrow \frac{y_{\mu}-t_{\mu}}{y_{\mu}-x_{\mu}} x_{v}+\frac{t_{\mu}-x_{\mu}}{y_{\mu}-x_{\mu}} y_{v}=t_{v} \\
\quad \frac{w_{v}-t_{v}}{w_{v}-z_{v}} z_{\mu}+\frac{t_{v}-z_{v}}{w_{v}-z_{v}} w_{\mu}=t_{\mu} \\
\rightarrow \frac{y_{\mu} x_{v}-t_{\mu} x_{v}+t_{\mu} y_{v}-x_{\mu} y_{v}}{y_{\mu}-x_{\mu}}=t_{v} \\
\frac{w_{v} z_{\mu}-t_{v} z_{\mu}+t_{v} w_{\mu}-z_{v} w_{\mu}}{w_{v}-z_{v}}=t_{\mu} \\
\rightarrow \frac{t_{\mu}\left(y_{v}-x_{v}\right)+y_{\mu} x_{v}-x_{\mu} y_{v}}{y_{\mu}-x_{\mu}}=t_{v} \\
\frac{t_{v}\left(w_{\mu}-z_{\mu}\right)+w_{v} z_{\mu}-z_{v} w_{\mu}}{w_{v}-z_{v}}=t_{\mu}
\end{gathered}
$$




$$
\begin{aligned}
& \rightarrow t_{\mu} \frac{\left(y_{v}-x_{v}\right)}{y_{\mu}-x_{\mu}}+\frac{y_{\mu} x_{v}-x_{\mu} y_{v}}{y_{\mu}-x_{\mu}}=t_{v} \\
& t_{v} \frac{\left(w_{\mu}-z_{\mu}\right)}{w_{v}-z_{v}}+\frac{w_{v} z_{\mu}-z_{v} w_{\mu}}{w_{v}-z_{v}}=t_{\mu}
\end{aligned}
$$

Now by substituting $t_{v}$ from Equation (c) into Equation (d) we find:

$$
\begin{gathered}
\left(t_{\mu} \frac{(y v-x v)}{y_{\mu}-x_{\mu}}+\frac{y_{\mu} x_{v}-x_{\mu} y_{v}}{y_{\mu}-x_{\mu}}\right)\left(\frac{\left(w_{\mu}-z_{\mu}\right)}{w_{v}-z_{v}}\right)+\frac{w_{v} z_{\mu}-z_{v} w_{\mu}}{w_{v}-z_{v}}=t_{\mu} \\
\rightarrow t_{\mu} \frac{\left(y_{v}-x_{v}\right)\left(w_{\mu}-z_{\mu}\right)}{\left(y_{\mu}-x_{\mu}\right)\left(w_{v}-z_{v}\right)}+\frac{\left(y_{\mu} x_{v}-x_{\mu} y_{v}\right)\left(w_{\mu}-z_{\mu}\right)}{\left(y_{\mu}-x_{\mu}\right)\left(w_{v}-z_{v}\right)}+\frac{w_{v} z_{\mu}-z_{v} w_{\mu}}{w_{v}-z_{v}}=t_{\mu} \\
\rightarrow t_{\mu}\left(y_{v}-x_{v}\right)\left(w_{\mu}-z_{\mu}\right)+\left(y_{\mu} x_{v}-x_{\mu} y_{v}\right)\left(w_{\mu}-z_{\mu}\right)+\left(w_{v} z_{\mu}-z_{v} w_{\mu}\right)\left(y_{\mu}-x_{\mu}\right) \\
=t_{\mu}\left(w_{v}-z_{v}\right)\left(y_{\mu}-x_{\mu}\right) \\
\rightarrow t_{\mu}\left(w_{v}-z_{v}\right)\left(y_{\mu}-x_{\mu}\right)-t_{\mu}\left(y_{v}-x_{v}\right)\left(w_{\mu}-z_{\mu}\right) \\
=\left(y_{\mu} x_{v}-x_{\mu} y_{v}\right)\left(w_{\mu}-z_{\mu}\right)+\left(w_{v} z_{\mu}-z_{v} w_{\mu}\right)\left(y_{\mu}-x_{\mu}\right) \\
t_{\mu}=\frac{\left(y_{\mu} x_{v}-x_{\mu} y_{v}\right)\left(w_{\mu}-z_{\mu}\right)+\left(w_{v} z_{\mu}-z_{v} w_{\mu}\right)\left(y_{\mu}-x_{\mu}\right)}{\left(w_{v}-z_{v}\right)\left(y_{\mu}-x_{\mu}\right)-\left(y_{v}-x_{v}\right)\left(w_{\mu}-z_{\mu}\right)}
\end{gathered}
$$

Now from Equation (a):

$$
t_{\varphi}=\frac{y_{\mu}-t_{\mu}}{y_{\mu}-x_{\mu}} x_{\varphi}+\frac{t_{\mu}-x_{\mu}}{y_{\mu}-x_{\mu}} y_{\varphi}
$$

For all $\varphi \in\{1,2, \cdots, n\}$.

$$
\begin{aligned}
& \rightarrow t_{\varphi}=\frac{y_{\mu} x_{\varphi}-t_{\mu} x_{\varphi}+t_{\mu} y_{\varphi}-x_{\mu} y_{\varphi}}{y_{\mu}-x_{\mu}} \\
& \rightarrow t_{\varphi}=\frac{y_{\mu} x_{\varphi}-x_{\mu} y_{\varphi}}{y_{\mu}-x_{\mu}}+t_{\mu} \frac{y_{\varphi}-x_{\varphi}}{y_{\mu}-x_{\mu}}
\end{aligned}
$$

Now by substituting $t_{\mu}$ from Equation (e) into Equation (f) we find:

$$
\begin{array}{r}
t_{\varphi}=\frac{y_{\mu} x_{\varphi}-x_{\mu} y_{\varphi}}{y_{\mu}-x_{\mu}}+\frac{\left(y_{\mu} x_{v}-x_{\mu} y_{v}\right)\left(w_{\mu}-z_{\mu}\right)+\left(w_{v} z_{\mu}-z_{v} w_{\mu}\right)\left(y_{\mu}-x_{\mu}\right)}{\left(w_{v}-z_{v}\right)\left(y_{\mu}-x_{\mu}\right)-\left(y_{v}-x_{v}\right)\left(w_{\mu}-z_{\mu}\right)} \frac{y_{\varphi}-x_{\varphi}}{y_{\mu}-x_{\mu}} \\
\rightarrow t_{\varphi}=\frac{y_{\mu} x_{\varphi}-x_{\mu} y_{\varphi}}{y_{\mu}-x_{\mu}}+\frac{\left(y_{\mu} x_{v}-x_{\mu} y_{v}\right)\left(w_{\mu}-z_{\mu}\right)-\left(z_{v} w_{\mu}-w_{v} z_{\mu}\right)\left(y_{\mu}-x_{\mu}\right)}{\left(y_{\mu}-x_{\mu}\right)\left(w_{v}-z_{v}\right)-\left(w_{\mu}-z_{\mu}\right)\left(y_{v}-x_{v}\right)} \frac{y_{\varphi}-x_{\varphi}}{y_{\mu}-x_{\mu}} \\
\rightarrow t_{\varphi}=\frac{[\boldsymbol{x}, \boldsymbol{y}]_{\mu, \varphi}}{\boldsymbol{d}_{\mu}(\boldsymbol{x}, \boldsymbol{y})}+\frac{[\boldsymbol{x}, \boldsymbol{y}]_{\mu, v} \boldsymbol{d}_{\mu}(\mathbf{z}, \boldsymbol{w})-[\mathbf{z}, \boldsymbol{w}]_{\mu, v} \boldsymbol{d}_{\mu}(\boldsymbol{x}, \boldsymbol{y})}{[\boldsymbol{d}(\boldsymbol{x}, \boldsymbol{y}), \boldsymbol{d}(\mathbf{z}, \boldsymbol{w})]_{\mu, v}(\boldsymbol{x}, \boldsymbol{y})} \frac{\boldsymbol{d}_{\mu}(\boldsymbol{x}, \boldsymbol{y})}{\boldsymbol{d}_{\mu}} \\
\rightarrow t_{\varphi}=\frac{[\overrightarrow{\boldsymbol{d}}(\boldsymbol{x}, \boldsymbol{y}), \overrightarrow{\boldsymbol{d}}(\mathbf{z}, \boldsymbol{w})]_{\mu, v}[\boldsymbol{x}, \boldsymbol{y}]_{\mu, \varphi}+[\boldsymbol{x}, \boldsymbol{y}]_{\mu, \nu} \boldsymbol{d}_{\mu}(\mathbf{z}, \boldsymbol{w}) \boldsymbol{d}_{\varphi}(\boldsymbol{x}, \boldsymbol{y})-[\mathbf{z}, \boldsymbol{w}]_{\mu, \nu} \boldsymbol{d}_{\mu}(\boldsymbol{x}, \boldsymbol{y}) \boldsymbol{d}_{\varphi}(\boldsymbol{x}, \boldsymbol{y})}{[\overrightarrow{\boldsymbol{d}}(\boldsymbol{x}, \boldsymbol{y}), \overrightarrow{\boldsymbol{d}}(\mathbf{z}, \boldsymbol{w})]_{\mu, v} \boldsymbol{d}_{\mu}(\boldsymbol{x}, \boldsymbol{y})}
\end{array}
$$

For all $\varphi \in\{1,2, \cdots, n\}$ and all $\mu, v \in\{1,2, \cdots, n\}$ fulfill $x_{\mu} \neq y_{\mu}, \quad z_{v} \neq w_{v}$ and either $x_{v} \neq y_{v}$ or $z_{\mu} \neq w_{\mu}$. 
This proves the first part of the theorem, now to prove the second part we can use the first proved part as following:

$$
\begin{aligned}
& t_{\varphi}=\frac{[\vec{d}(x, y), \vec{d}(z, w)]_{\mu, v}[x, y]_{\mu, \varphi}+[x, y]_{\mu, v} d_{\mu}(z, w) d_{\varphi}(x, y)-[z, w]_{\mu, v} d_{\mu}(x, y) d_{\varphi}(x, y)}{[\vec{d}(x, y), \vec{d}(z, w)]_{\mu, \nu} d_{\mu}(x, y)} \\
& {[\vec{d}(x, y), \vec{d}(z, w)]_{\mu, v} d_{\mu}(x, y) t_{\varphi}} \\
& =[\vec{d}(x, y), \vec{d}(z, w)]_{\mu, v}[x, y]_{\mu, \varphi}+[x, y]_{\mu, v} d_{\mu}(z, w) d_{\varphi}(x, y)-[z, w]_{\mu, v} d_{\mu}(x, y) d_{\varphi}(x, y) \\
& \rightarrow[\vec{d}(x, y), \vec{d}(z, w)]_{\mu, v} d_{\mu}(x, y) t_{\varphi}-[\vec{d}(x, y), \vec{d}(z, w)]_{\mu, v}[x, y]_{\mu, \varphi} \\
& =[x, y]_{\mu, \nu} d_{\mu}(z, w) d_{\varphi}(x, y)-[z, w]_{\mu, v} d_{\mu}(x, y) d_{\varphi}(x, y) \\
& \rightarrow[\vec{d}(x, y), \vec{d}(z, w)]_{\mu, v}\left(t_{\varphi} d_{\mu}(x, y)-[x, y]_{\mu, \varphi}\right) \\
& =[x, y]_{\mu, v} d_{\mu}(z, w) d_{\varphi}(x, y)-[z, w]_{\mu, v} d_{\mu}(x, y) d_{\varphi}(x, y)
\end{aligned}
$$

Thus if $\boldsymbol{x}=\boldsymbol{t}+a \overrightarrow{\boldsymbol{d t}}, \boldsymbol{y}=\boldsymbol{t}+b \overrightarrow{\boldsymbol{d t}}, \mathbf{z}=\boldsymbol{t}+a^{\prime} \overrightarrow{\boldsymbol{d t ^ { \prime }}}$ and $\boldsymbol{w}=\boldsymbol{t}+b^{\prime} \overrightarrow{\boldsymbol{d t ^ { \prime }}}$ for some $a, b, a^{\prime}, b^{\prime} \in \mathbb{R} /\{0\}$ fulfill $a \neq b$ and $a^{\prime} \neq b^{\prime}$, and some $\overrightarrow{\boldsymbol{d} t}=\left[\begin{array}{c}d t_{1} \\ d t_{2} \\ \vdots \\ d t_{n}\end{array}\right] \in \mathbb{R}^{n}$ and $\overrightarrow{\boldsymbol{d t}^{\prime}}=\left[\begin{array}{c}d t_{1}^{\prime} \\ d t_{2}^{\prime} \\ \vdots \\ d t_{n}^{\prime}\end{array}\right]$ fulfill $d t_{\mu} \neq 0, \quad d t_{v}^{\prime} \neq 0, \overrightarrow{\mathbf{d t}^{\prime}} \neq \lambda \overrightarrow{\boldsymbol{d t}}$ for all $\lambda \in \mathbb{R}-i . e . \overrightarrow{\mathbf{d t}^{\prime}}$ is not parallel to $\overrightarrow{\boldsymbol{d t}}$-and either $d t_{v} \neq 0$ or $d t_{\mu}^{\prime} \neq 0$, then:

$$
\begin{aligned}
& {\left[(b-a) \overrightarrow{\boldsymbol{d} t},\left(b^{\prime}-a^{\prime}\right) \overrightarrow{\boldsymbol{d} \boldsymbol{t}^{\prime}}\right]_{\mu, \nu}\left(t_{\varphi}(b-a) d t_{\mu}-[\boldsymbol{t}+a \overrightarrow{\boldsymbol{d} t}, \boldsymbol{t}+b \overrightarrow{\boldsymbol{d} \boldsymbol{t}}]_{\mu, \varphi}\right)} \\
& =[\boldsymbol{t}+a \overrightarrow{\boldsymbol{d} t}, \boldsymbol{t}+b \overrightarrow{\mathbf{d} \boldsymbol{t}}]_{\mu, v}\left(b^{\prime}-a^{\prime}\right) d t_{\mu}^{\prime}(b-a) d t_{\varphi} \\
& -\left[\boldsymbol{t}+a^{\prime} \overrightarrow{\mathbf{d t}^{\prime}}, \boldsymbol{t}+b^{\prime} \overrightarrow{\mathbf{d t}^{\prime}}\right]_{\mu, v}(b-a) d t_{\mu}(b-a) d t_{\varphi} \\
& (b-a)\left(b^{\prime}-a^{\prime}\right)\left[\overrightarrow{\mathbf{d} t}, \overrightarrow{\boldsymbol{d} \boldsymbol{t}^{\prime}}\right]_{\mu, \nu}\left(t_{\varphi}(b-a) d t_{\mu}-(b-a)[\boldsymbol{t}, \overrightarrow{\boldsymbol{d} t}]_{\mu, \varphi}\right) \\
& \rightarrow=(b-a)[\boldsymbol{t}, \overrightarrow{\boldsymbol{d} t}]_{\mu, v}\left(b^{\prime}-a^{\prime}\right) d t_{\mu}^{\prime}(b-a) d t_{\varphi} \\
& -\left(b^{\prime}-a^{\prime}\right)\left[\boldsymbol{t}, \overrightarrow{\boldsymbol{d t}^{\prime}}\right]_{\mu, v}(b-a) d t_{\mu}(b-a) d t_{\varphi} \\
& \rightarrow\left[\overrightarrow{\boldsymbol{d} t}, \overrightarrow{\boldsymbol{d t}^{\prime}}\right]_{\mu, \nu}\left(t_{\varphi} d t_{\mu}-[\boldsymbol{t}, \overrightarrow{\boldsymbol{d} t}]_{\mu, \varphi}\right)=[\boldsymbol{t}, \overrightarrow{\boldsymbol{d} t}]_{\mu, \nu} d t_{\mu}^{\prime} d t_{\varphi}-\left[\boldsymbol{t}, \overrightarrow{\boldsymbol{d t}^{\prime}}\right]_{\mu, \nu} d t_{\mu} d t_{\varphi}
\end{aligned}
$$

For all $\varphi \in\{1,2, \cdots, n\}$ and all $\mu, v \in\{1,2, \cdots, n\}$ fulfill $d t_{\mu} \neq 0, d t_{v}^{\prime} \neq 0$ and either $d t_{v} \neq 0$ or $d t_{\mu}^{\prime} \neq 0$.

This equation is representing the fundamental fluctuation tensor field equations which are invariant under any arbitrary change of $\boldsymbol{t}, \overrightarrow{\boldsymbol{d} t}$ and $\overrightarrow{\boldsymbol{d t}^{\prime}}$ by any $\boldsymbol{\tau}=\left(\tau_{1}, \tau_{2}, \cdots, \tau_{n}\right) \in \mathbb{R}^{n}, \quad \overrightarrow{\boldsymbol{d} \tau}=\left[\begin{array}{c}d \tau_{1} \\ d \tau_{2} \\ \vdots \\ d \tau_{n}\end{array}\right] \in \mathbb{R}^{n}$ and $\overrightarrow{\boldsymbol{d} \tau^{\prime}}=\left[\begin{array}{c}d \tau_{1}^{\prime} \\ d \tau_{2}^{\prime} \\ \vdots \\ d \tau_{n}^{\prime}\end{array}\right] \in \mathbb{R}^{n}$ respectively 
such that $\overrightarrow{\boldsymbol{d} \tau^{\prime}} \neq \lambda \overrightarrow{\boldsymbol{d} \tau}$ for all $\lambda \in \mathbb{R}$, thus:

$$
\left[\overrightarrow{\boldsymbol{d} \tau}, \overrightarrow{\boldsymbol{d} \tau^{\prime}}\right]_{\mu, v}\left(\tau_{\varphi} d \tau_{\mu}-[\tau, \overrightarrow{\boldsymbol{d} \tau}]_{\mu, \varphi}\right)=[\tau, \overrightarrow{\boldsymbol{d} \tau}]_{\mu, \nu} d \tau_{\mu}^{\prime} d \tau_{\varphi}-\left[\tau, \overrightarrow{\boldsymbol{d} \tau^{\prime}}\right]_{\mu, \nu} d \tau_{\mu} d \tau_{\varphi}
$$

For all $\varphi \in\{1,2, \cdots, n\}$ and all $\mu, v \in\{1,2, \cdots, n\}$ fulfill $d \tau_{\mu} \neq 0, d \tau_{v}^{\prime} \neq 0$ and either $d \tau_{v} \neq 0$ or $d \tau_{\mu}^{\prime} \neq 0$.

\subsection{Orthogonal n-Dimensional Real Collinear Sets}

For each $\boldsymbol{x}, \boldsymbol{y}, \boldsymbol{z}, \boldsymbol{w} \in \mathbb{R}^{n}$ the two collinear sets $\mathbb{R}^{n}(\boldsymbol{x}, \boldsymbol{y})$ and $\mathbb{R}^{n}(\mathbf{z}, \boldsymbol{w})$ are called orthogonal if and only if:

1) $\mathbb{R}^{n}(\boldsymbol{x}, \boldsymbol{y}) \cap \mathbb{R}^{n}(\mathbf{z}, \boldsymbol{w}) \neq\{\varnothing\}$.

2) $\overrightarrow{\boldsymbol{d}}(\boldsymbol{a}, \boldsymbol{b}) \cdot \overrightarrow{\boldsymbol{d}}(\boldsymbol{e}, \boldsymbol{d})=0$, for all $\boldsymbol{a}, \boldsymbol{b} \in \mathbb{R}^{n}(\boldsymbol{x}, \boldsymbol{y})$ and $\boldsymbol{d}, \boldsymbol{e} \in \mathbb{R}^{n}(\mathbf{z}, \boldsymbol{w})$.

Such that $(\boldsymbol{b}-\boldsymbol{a}) \cdot(\boldsymbol{d}-\boldsymbol{e})$ donate to the dot product of $(\boldsymbol{b}-\boldsymbol{a})$ and $(\boldsymbol{d}-\boldsymbol{e})$.

\subsection{N-Dimensional Real Collinear Sets Space}

The n-dimensional real collinear sets local space at $t \in \mathbb{R}^{n}$ donated by $\mathbb{R}^{n}[\boldsymbol{t}]$ is the space of all $\mathrm{n}$-dimensional real collinear sets intersected at the point $t$, which is given as following:

$$
\mathbb{R}^{n}[\boldsymbol{t}]=\left\{\mathbb{R}^{n}(\boldsymbol{t}+a \overrightarrow{\boldsymbol{d} t}, \boldsymbol{t}+b \overrightarrow{\boldsymbol{d} \boldsymbol{t}}) \mid \overrightarrow{\boldsymbol{d} \boldsymbol{t}} \in \mathbb{R}^{n} /\{0\} \text { and } a, b \in \mathbb{R} /\{0\} \text { fulfill } a \neq b\right\} .
$$

\section{Important note}

Any $\boldsymbol{t}=\left(t_{1}, t_{2}, \cdots, t_{n}\right) \in \mathbb{R}^{n}$ is representing the origin of $\mathbb{R}^{n}[\boldsymbol{t}]$ fulfills for any two collinear sets $\mathbb{R}^{n}(\boldsymbol{t}+a \overrightarrow{\boldsymbol{d} t}, \boldsymbol{t}+b \overrightarrow{\boldsymbol{d} \boldsymbol{t}})$ and $\mathbb{R}^{n}\left(\boldsymbol{t}+a^{\prime} \overrightarrow{\boldsymbol{d t}^{\prime}}, \boldsymbol{t}+b^{\prime} \overrightarrow{\boldsymbol{d t}^{\prime}}\right)$ belong to $\mathbb{R}^{n}[\boldsymbol{t}]$, such that $\overrightarrow{\boldsymbol{d}}=\left[\begin{array}{c}d t_{1} \\ d t_{2} \\ \vdots \\ d t_{n}\end{array}\right] \in \mathbb{R}^{n}, \overrightarrow{\mathbf{d t}^{\prime}}=\left[\begin{array}{c}d t_{1}^{\prime} \\ d t_{2}^{\prime} \\ \vdots \\ d t_{n}^{\prime}\end{array}\right] \in \mathbb{R}^{n}$, fulfill $\overrightarrow{\boldsymbol{d t}^{\prime}} \neq \lambda \overrightarrow{\boldsymbol{d} \boldsymbol{t}}$ for all $\lambda \in \mathbb{R}$-i.e. $\overrightarrow{\mathbf{d t}^{\prime}}$ is not parallel to $\overrightarrow{\boldsymbol{d t}}$-and $a, b, a^{\prime}, b^{\prime} \in \mathbb{R} /\{0\}$ fulfill $a \neq b$ and $a^{\prime} \neq b^{\prime}$ the equations:

$$
\left[\overrightarrow{\mathbf{d}}, \overrightarrow{\boldsymbol{d t}^{\prime}}\right]_{\mu, \nu}\left(t_{\varphi} d t_{\mu}-[\boldsymbol{t}, \overrightarrow{\boldsymbol{d} t}]_{\mu, \varphi}\right)=[\boldsymbol{t}, \overrightarrow{\boldsymbol{d} t}]_{\mu, \nu} d t_{\mu}^{\prime} d t_{\varphi}-[\boldsymbol{t}, \overrightarrow{\boldsymbol{d} t}]_{\mu, \nu} d t_{\mu} d t_{\varphi}
$$

For all $\varphi \in\{1,2, \cdots, n\}$ and all $\mu, v \in\{1,2, \cdots, n\}$ fulfills $d t_{\mu} \neq 0, d t_{v}^{\prime} \neq 0$ and either $d t_{v} \neq 0$ or $d t_{\mu}^{\prime} \neq 0$, these equations are invariant under any arbitrary change of $\overrightarrow{d t}$ and $\overrightarrow{d t^{\prime}}$.

\subsection{The n-Dimensional Real Collinear Sets Bundle}

The $\mathrm{n}$-dimensional real collinear sets bundle is the union of all $\mathrm{n}$-dimensional real collinear sets spaces at all points in $\mathbb{R}^{n}$ which is given as:

$$
\mathbb{R}^{[n]}=\bigcup_{t \in \mathbb{R}^{n}}\left(\mathbb{R}^{n}[\boldsymbol{t}]\right)
$$

\subsection{The n-Dimensional Real Coplanar Set}

For all $\boldsymbol{p}, \boldsymbol{q}, \boldsymbol{r} \in \mathbb{R}^{\mathrm{n}}$ the $\mathrm{n}$-dimensional coplanar set of $\boldsymbol{p}, \boldsymbol{q}$ and $\boldsymbol{r}$ donated by $\mathbb{R}^{n}(\boldsymbol{p}, \boldsymbol{q}, \boldsymbol{r})$ is a set of all point in $\mathbb{R}^{\mathrm{n}}$ lied at the $\mathrm{n}$-dimensional plane contains 
$p$, $\boldsymbol{q}$ and $\boldsymbol{r}$, which is defined as following:

$$
\begin{aligned}
\mathbb{R}^{n}(\boldsymbol{p}, \boldsymbol{q}, \boldsymbol{r}) & =\left\{\mathbb{R}^{n}(\boldsymbol{p}+x \overrightarrow{\boldsymbol{d}}(\boldsymbol{q}, \boldsymbol{r}), \boldsymbol{q}+x \overrightarrow{\boldsymbol{d}}(\boldsymbol{q}, \boldsymbol{r})) \mid \overrightarrow{\boldsymbol{d}}(\boldsymbol{q}, \boldsymbol{r})=\boldsymbol{r}-\boldsymbol{q} \text { and } x \in \mathbb{R}\right\} \\
& =\left\{\mathbb{R}^{n}(\boldsymbol{q}+y \overrightarrow{\boldsymbol{d}}(\boldsymbol{r}, \boldsymbol{p}), \boldsymbol{r}+y \overrightarrow{\boldsymbol{d}}(\boldsymbol{r}, \boldsymbol{p})) \mid \overrightarrow{\boldsymbol{d}}(\boldsymbol{r}, \boldsymbol{p})=\boldsymbol{p}-\boldsymbol{r} \text { and } y \in \mathbb{R}\right\} \\
& =\left\{\mathbb{R}^{n}(\boldsymbol{r}+z \overrightarrow{\boldsymbol{d}}(\boldsymbol{p}, \boldsymbol{q}), \boldsymbol{p}+z \overrightarrow{\boldsymbol{d}}(\boldsymbol{p}, \boldsymbol{q})) \mid \overrightarrow{\boldsymbol{d}}(\boldsymbol{p}, \boldsymbol{q})=\boldsymbol{q}-\boldsymbol{p} \text { and } z \in \mathbb{R}\right\} .
\end{aligned}
$$

\subsection{The n-Dimensional Real Coplanar Space}

The $\mathrm{n}$-dimensional real coplanar space at each point $(x, y, z) \in \mathbb{R}^{3}$ donated by $\mathbb{R}^{n}[x, y, z]$ is defined as following:

$$
\begin{aligned}
\mathbb{R}^{n}[x, y, z]= & \left\{\mathbb{R}^{n}(\boldsymbol{p}, \boldsymbol{q}, \boldsymbol{r}) \mid \boldsymbol{p}, \boldsymbol{q}, \boldsymbol{r} \in \mathbb{R}^{n} \text { fulfill } \mathbb{R}^{n}(\boldsymbol{p}+x \overrightarrow{\boldsymbol{d}}(\boldsymbol{q}, \boldsymbol{r}), \boldsymbol{q}+x \overrightarrow{\boldsymbol{d}}(\boldsymbol{q}, \boldsymbol{r}))\right. \\
& \bigcap \mathbb{R}^{n}(\boldsymbol{q}+y \overrightarrow{\boldsymbol{d}}(\boldsymbol{r}, \boldsymbol{p}), \boldsymbol{r}+y \overrightarrow{\boldsymbol{d}}(\boldsymbol{r}, \boldsymbol{p}))=\mathbb{R}^{n}(\boldsymbol{q}+y \overrightarrow{\boldsymbol{d}}(\boldsymbol{r}, \boldsymbol{p}), \boldsymbol{r}+y \overrightarrow{\boldsymbol{d}}(\boldsymbol{r}, \boldsymbol{p})) \\
& \bigcap \mathbb{R}^{n}(\boldsymbol{p}+z \overrightarrow{\boldsymbol{d}}(\boldsymbol{p}, \boldsymbol{q}), \boldsymbol{r}+z \overrightarrow{\boldsymbol{d}}(\boldsymbol{p}, \boldsymbol{q}))=\mathbb{R}^{n}(\boldsymbol{p}+x \overrightarrow{\boldsymbol{d}}(\boldsymbol{q}, \boldsymbol{r}), \boldsymbol{q}+x \overrightarrow{\boldsymbol{d}}(\boldsymbol{q}, \boldsymbol{r})) \\
& \left.\bigcap \mathbb{R}^{n}(\boldsymbol{p}+z \overrightarrow{\boldsymbol{d}}(\boldsymbol{p}, \boldsymbol{q}), \boldsymbol{r}+z \overrightarrow{\boldsymbol{d}}(\boldsymbol{p}, \boldsymbol{q}))\right\}
\end{aligned}
$$

\section{Important notes:}

$\mathbb{R}^{n}[x, y, z]$ is defined as the space of all coplanars $\mathbb{R}^{n}(\boldsymbol{p}, \boldsymbol{q}, \boldsymbol{r})$ that are containing some point $\boldsymbol{t}=\left(t_{1}, t_{2}, \cdots, t_{n}\right) \in \mathbb{R}^{n}(\boldsymbol{p}, \boldsymbol{q}, \boldsymbol{r})$ represents intersection point of collinear sets then using the theorem (3.6) we find:

$$
\begin{aligned}
t_{\varphi}= & \frac{[\boldsymbol{p}+x \overrightarrow{\boldsymbol{d}}(\boldsymbol{q}, \boldsymbol{r}), \boldsymbol{q}+x \overrightarrow{\boldsymbol{d}}(\boldsymbol{q}, \boldsymbol{r})]_{\mu, \varphi}}{\boldsymbol{d}_{\mu}(\boldsymbol{p}, \boldsymbol{q})}+\frac{[\boldsymbol{p}+x \overrightarrow{\boldsymbol{d}}(\boldsymbol{q}, \boldsymbol{r}), \boldsymbol{q}+x \overrightarrow{\boldsymbol{d}}(\boldsymbol{q}, \boldsymbol{r})]_{\mu, v} \boldsymbol{d}_{\mu}(\boldsymbol{q}, \boldsymbol{r}) \boldsymbol{d}_{\varphi}(\boldsymbol{p}, \boldsymbol{q})}{[\overrightarrow{\boldsymbol{d}}(\boldsymbol{p}, \boldsymbol{q}), \overrightarrow{\boldsymbol{d}}(\boldsymbol{q}, \boldsymbol{r})]_{\mu, v} \boldsymbol{d}_{\mu}(\boldsymbol{p}, \boldsymbol{q})} \\
& -\frac{[\boldsymbol{q}+y \overrightarrow{\boldsymbol{d}}(\boldsymbol{r}, \boldsymbol{p}), \boldsymbol{r}+y \overrightarrow{\boldsymbol{d}}(\boldsymbol{r}, \boldsymbol{p})]_{\mu, v} \boldsymbol{d}_{\varphi}(\boldsymbol{p}, \boldsymbol{q})}{[\overrightarrow{\boldsymbol{d}}(\boldsymbol{p}, \boldsymbol{q}), \overrightarrow{\boldsymbol{d}}(\boldsymbol{q}, \boldsymbol{r})]_{\mu, v}} \\
= & \frac{[\boldsymbol{q}+y \overrightarrow{\boldsymbol{d}}(\boldsymbol{r}, \boldsymbol{p}), \boldsymbol{r}+y \overrightarrow{\boldsymbol{d}}(\boldsymbol{r}, \boldsymbol{p})]_{v, \varphi}}{\boldsymbol{d}_{v}(q, r)}+\frac{[\boldsymbol{q}+y \overrightarrow{\boldsymbol{d}}(\boldsymbol{r}, \boldsymbol{p}), \boldsymbol{r}+y \overrightarrow{\boldsymbol{d}}(\boldsymbol{r}, \boldsymbol{p})]_{v, \alpha} \boldsymbol{d}_{v}(\boldsymbol{r}, \boldsymbol{p}) \boldsymbol{d}_{\varphi}(\boldsymbol{q}, \boldsymbol{r})}{[\overrightarrow{\boldsymbol{d}}(\boldsymbol{q}, \boldsymbol{r}), \overrightarrow{\boldsymbol{d}}(\boldsymbol{r}, \boldsymbol{p})]_{v, \alpha} \boldsymbol{d}_{v}(\boldsymbol{q}, \boldsymbol{r})} \\
& -\frac{[\boldsymbol{r}+z \overrightarrow{\boldsymbol{d}}(\boldsymbol{p}, \boldsymbol{q}), \boldsymbol{p}+z \overrightarrow{\boldsymbol{d}}(\boldsymbol{p}, \boldsymbol{q})]_{v, \alpha} \boldsymbol{d}_{\varphi}(\boldsymbol{q}, \boldsymbol{r})}{[\overrightarrow{\boldsymbol{d}}(\boldsymbol{q}, \boldsymbol{r}), \overrightarrow{\boldsymbol{d}}(\boldsymbol{r}, \boldsymbol{p})]_{v, \alpha}} \\
= & \frac{[\boldsymbol{r}+z \overrightarrow{\boldsymbol{d}}(\boldsymbol{p}, \boldsymbol{q}), \boldsymbol{p}+z \overrightarrow{\boldsymbol{d}}(\boldsymbol{p}, \boldsymbol{q})]_{\alpha, \varphi}}{\boldsymbol{d}(\boldsymbol{r}, \boldsymbol{p})}+\frac{[\boldsymbol{r}+z \overrightarrow{\boldsymbol{d}}(\boldsymbol{p}, \boldsymbol{q}), \boldsymbol{p}+z \overrightarrow{\boldsymbol{d}}(\boldsymbol{p}, \boldsymbol{q})]_{\alpha, \mu} \boldsymbol{d}_{\alpha}(\boldsymbol{p}, \boldsymbol{q}) \boldsymbol{d}_{\varphi}(\boldsymbol{r}, \boldsymbol{p})}{[\overrightarrow{\boldsymbol{d}}(\boldsymbol{r}, \boldsymbol{p}), \overrightarrow{\boldsymbol{d}}(\boldsymbol{p}, \boldsymbol{q})]_{\alpha, \mu} \boldsymbol{d}_{\alpha}(\boldsymbol{r}, \boldsymbol{p})} \\
& -\frac{[\boldsymbol{p}+x \overrightarrow{\boldsymbol{d}}(q, r), \boldsymbol{q}+x \overrightarrow{\boldsymbol{d}}(q, r)]_{\alpha, \mu} \boldsymbol{d}_{\varphi}(\boldsymbol{r}, \boldsymbol{p})}{[\overrightarrow{\boldsymbol{d}}(\boldsymbol{r}, \boldsymbol{p}), \overrightarrow{\boldsymbol{d}}(\boldsymbol{p}, \boldsymbol{q})]_{\alpha, \mu}}
\end{aligned}
$$

For all $\varphi \in\{1,2, \cdots, n\}$ and all $\mu, v, \alpha \in\{1,2, \cdots, n\}$ fulfills $[\vec{d}(\boldsymbol{p}, \boldsymbol{q}), \overrightarrow{\boldsymbol{d}}(\boldsymbol{q}, \boldsymbol{r})]_{\mu, v} \boldsymbol{d}_{\mu}(\boldsymbol{p}, \boldsymbol{q}) \neq 0,[\overrightarrow{\boldsymbol{d}}(\boldsymbol{q}, \boldsymbol{r}), \overrightarrow{\boldsymbol{d}}(\boldsymbol{r}, \boldsymbol{p})]_{v, \alpha} \boldsymbol{d}_{v}(\boldsymbol{q}, \boldsymbol{r}) \neq 0$ and $[\vec{d}(r, p), \vec{d}(p, q)]_{\alpha, \mu} d_{\alpha}(r, p) \neq 0$.

\subsection{The n-Dimensional Real Coplanar Bundle}

The n-dimensional real coplanar bundle is union of all n-dimensional real cop- 
lanar spaces at all space point in $\mathbb{R}^{3}$ which is defined as following:

$$
\mathbb{R}^{n}\left[\mathbb{R}^{3}\right]=\bigcup_{(x, y, z) \in \mathbb{R}^{3}}\left(\mathbb{R}^{n}[x, y, z]\right)
$$

\subsection{The N-Dimensional Real Space-Time}

For any physical system consisting of $\mathrm{N}$ matter particles, the $\mathrm{N}$-dimensional real space-time is the section of $\mathrm{N}$-dimensional real coplanar bundle that consisting of all real-time states of the physical systems at each all space point occupied by one or more constituent matter particles of the physical system during finite epoch with respect to an arbitrary observer. Thus if $\mathbb{R}^{+N}=\{r \in \mathbb{R} \mid r \geq 0\}$ then at each space point $(x, y, z) \in \mathbb{R}^{3}$ occupied by one or more constituent matter particles of the physical system the real-time state of the physical system is representing an element of the section of $\mathbb{R}^{N}[x, y, z]$ that defined as following:

$$
\begin{aligned}
\mathbb{R}^{\mathrm{ST}}[x, y, z]=\{ & \mathbb{R}^{N}\left(\boldsymbol{P}_{a}, \boldsymbol{P}_{r}, \boldsymbol{F}_{u}\right) \mid \boldsymbol{P}_{a}, \boldsymbol{P}_{r}, \boldsymbol{F}_{u} \in \mathbb{R}^{+N} \text { fulfill } \\
& \mathbb{R}^{N}\left(\boldsymbol{P}_{a}+x \overrightarrow{\boldsymbol{d}}\left(\boldsymbol{P}_{r}, \boldsymbol{F}_{u}\right), \boldsymbol{P}_{r}+x \overrightarrow{\boldsymbol{d}}\left(\boldsymbol{P}_{r}, \boldsymbol{F}_{u}\right)\right) \\
& \cap \mathbb{R}^{N}\left(\boldsymbol{P}_{r}+y \overrightarrow{\boldsymbol{d}}\left(\boldsymbol{F}_{u}, \boldsymbol{P}_{a}\right), \boldsymbol{F}_{u}+y \overrightarrow{\boldsymbol{d}}\left(\boldsymbol{F}_{u}, \boldsymbol{P}_{a}\right)\right) \\
& =\mathbb{R}^{N}\left(\boldsymbol{P}_{r}+y \overrightarrow{\boldsymbol{d}}\left(\boldsymbol{F}_{u}, \boldsymbol{P}_{a}\right), \boldsymbol{F}_{u}+y \overrightarrow{\boldsymbol{d}}\left(\boldsymbol{F}_{u}, \boldsymbol{P}_{a}\right)\right) \\
& \cap \mathbb{R}^{n}\left(\boldsymbol{P}_{a}+z \overrightarrow{\boldsymbol{d}}\left(\boldsymbol{P}_{a}, \boldsymbol{P}_{r}\right), \boldsymbol{F}_{u}+z \overrightarrow{\boldsymbol{d}}\left(\boldsymbol{P}_{a}, \boldsymbol{P}_{r}\right)\right) \\
& =\mathbb{R}^{N}\left(\boldsymbol{P}_{a}+x \overrightarrow{\boldsymbol{d}}\left(\boldsymbol{P}_{r}, \boldsymbol{F}_{u}\right), \boldsymbol{P}_{r}+x \overrightarrow{\boldsymbol{d}}\left(\boldsymbol{P}_{r}, \boldsymbol{F}_{u}\right)\right) \\
& \left.\cap \mathbb{R}^{N}\left(\boldsymbol{P}_{a}+z \overrightarrow{\boldsymbol{d}}\left(\boldsymbol{P}_{a}, \boldsymbol{P}_{r}\right), \boldsymbol{F}_{u}+z \overrightarrow{\boldsymbol{d}}\left(\boldsymbol{P}_{a}, \boldsymbol{P}_{r}\right)\right)\right\}
\end{aligned}
$$

\section{Important notes:}

If the real-time state of the physical system at the space point $(x, y, z) \in \mathbb{R}^{3}$ occupied by one or more constituent matter particles of it is defined according to the equation 9 as following:

$$
\begin{aligned}
\left|t\left(x, y, z, n_{1}, n_{2}, \cdots, n_{N}\right)\right\rangle= & \left|P_{a}\left(x, y, z, n_{1}, n_{2}, \cdots, n_{N}\right)\right\rangle \\
& +\left|P_{r}\left(x, y, z, n_{1}, n_{2}, \cdots, n_{N}\right)\right\rangle+\left|F_{u}\left(x, y, z, n_{1}, n_{2}, \cdots, n_{N}\right)\right\rangle
\end{aligned},
$$

then there exist at least in principle $\boldsymbol{P}_{a}, \boldsymbol{P}_{r}, \boldsymbol{F}_{u} \in \mathbb{R}^{+N}$ fulfill

$$
\begin{aligned}
& \overrightarrow{\boldsymbol{P}_{a}}=\left|P_{a}\left(x, y, z, n_{1}, n_{2}, \cdots, n_{N}\right)\right\rangle, \quad \overrightarrow{\boldsymbol{P}_{r}}=\left|P_{r}\left(x, y, z, n_{1}, n_{2}, \cdots, n_{N}\right)\right\rangle, \\
& \overrightarrow{\boldsymbol{F}_{u}}=\left|F_{u}\left(x, y, z, n_{1}, n_{2}, \cdots, n_{N}\right)\right\rangle \text { and } \\
& \left|t\left(x, y, z, n_{1}, n_{2}, \cdots, n_{N}\right)\right\rangle \\
& =\mathbb{R}^{N}\left(\boldsymbol{P}_{a}+x \overrightarrow{\boldsymbol{d}}\left(\boldsymbol{P}_{r}, \boldsymbol{F}_{u}\right), \boldsymbol{P}_{r}+x \overrightarrow{\boldsymbol{d}}\left(\boldsymbol{P}_{r}, \boldsymbol{F}_{u}\right)\right) \\
& \cap \mathbb{R}^{N}\left(\boldsymbol{P}_{r}+y \vec{d}\left(\boldsymbol{F}_{u}, \boldsymbol{P}_{a}\right), \boldsymbol{F}_{u}+y \overrightarrow{\boldsymbol{d}}\left(\boldsymbol{F}_{u}, \boldsymbol{P}_{a}\right)\right) \\
& =\mathbb{R}^{N}\left(\boldsymbol{P}_{r}+y \vec{d}\left(\boldsymbol{F}_{u}, \boldsymbol{P}_{a}\right), \boldsymbol{F}_{u}+y \overrightarrow{\boldsymbol{d}}\left(\boldsymbol{F}_{u}, \boldsymbol{P}_{a}\right)\right) \\
& \cap \mathbb{R}^{n}\left(\boldsymbol{P}_{a}+z \overrightarrow{\boldsymbol{d}}\left(\boldsymbol{P}_{a}, \boldsymbol{P}_{r}\right), \boldsymbol{F}_{u}+z \overrightarrow{\boldsymbol{d}}\left(\boldsymbol{P}_{a}, \boldsymbol{P}_{r}\right)\right) \\
& =\mathbb{R}^{N}\left(\boldsymbol{P}_{a}+x \overrightarrow{\boldsymbol{d}}\left(\boldsymbol{P}_{r}, \boldsymbol{F}_{u}\right), \boldsymbol{P}_{r}+x \overrightarrow{\boldsymbol{d}}\left(\boldsymbol{P}_{r}, \boldsymbol{F}_{u}\right)\right) \\
& \cap \mathbb{R}^{N}\left(\boldsymbol{P}_{a}+z \overrightarrow{\boldsymbol{d}}\left(\boldsymbol{P}_{a}, \boldsymbol{P}_{r}\right), \boldsymbol{F}_{u}+z \overrightarrow{\boldsymbol{d}}\left(\boldsymbol{P}_{a}, \boldsymbol{P}_{r}\right)\right)
\end{aligned}
$$

\section{Conclusion}

As the spatial coordinates $x, y$ and $z$ which are representing the lengths between 
origin $(0,0,0)$ and $(x, 0,0),(0, y, 0)$ and $(0,0, z)$ respectively, the time coordinate is representing the total length of all occupation and leaving epochs of space point including the length of the initial leaving epoch elapsed before the first occupation of space point by the matter particle during finite observation epoch, this implied that the direction of time of any matter particle at each space point $P$ occupied by it is the direction of increasing the number of occupation and leaving epochs of $P$ by it, and the measurement of occupation of space point by one constituent matter particles of the physical system produce the same measurement of the time of the rest matter particles of the physical system that occupied and left the space point during finite observation epoch regardless of their distribution in space, this give simple reinterpretation of quantum entanglement. The motion of the constituent matter particles on separated orbits is equivalent to their motion in separated digital levels, and their transitions from one orbit to another one is equivalent to their transition from one digital level to another.

\section{Acknowledgements}

Thanks for my father who supported all my education levels and for my wife Ayaat Ahmed Osman for here incorporeal support for me in scientific papers publications.

\section{References}

[1] Einstein (1916) The Foundation of the Generalized Theory of Relativity. https://en.wikisource.org/wiki/The_Foundation_of_the_Generalised_Theory_of_Re lativity

[2] Einstein, Podolsky and Rosen (1935) Can Quantum-Mechanical Description of Physical Reality Be Considered Complete?

[3] Bell, J. (1964) Physics, 1, 195-200.

[4] McTaggard, J.E. (1908) The Unreality of Time. https://docs.google.com/viewer?a=v\&pid=sites\&srcid=ZGVmYXVsdGRvbWFpbnx 0eXNnc210ZXxneDozYmE1OWUyMGU0NjU1YzE4

[5] Quantum Experiment Shows How Time “Emerges” from Entanglement. https://medium.com/the-physics-arxiv-blog/quantum-experiment-shows-how-time -emerges-from-entanglement-d5d3dc850933\#.733j5zlez

[6] New Quantum Theory Could Explain the Flow of Time. WIRED. Retrieved 13 October 2014. 
Submit or recommend next manuscript to SCIRP and we will provide best service for you:

Accepting pre-submission inquiries through Email, Facebook, LinkedIn, Twitter, etc. A wide selection of journals (inclusive of 9 subjects, more than 200 journals)

Providing 24-hour high-quality service

User-friendly online submission system

Fair and swift peer-review system

Efficient typesetting and proofreading procedure

Display of the result of downloads and visits, as well as the number of cited articles Maximum dissemination of your research work

Submit your manuscript at: http://papersubmission.scirp.org/

Or contact jmp@scirp.org 